\title{
Higgs-Mediated Optical Amplification in a Nonequilibrium Superconductor
}

\author{
Michele Buzzi®, ${ }^{1, *}$ Gregor Jotzu $\odot,{ }^{1, *}$ Andrea Cavalleri $\odot,{ }^{1}$ J. Ignacio Cirac, ${ }^{2}$ Eugene A. Demler, \\ Bertrand I. Halperin $\odot,{ }^{3}$ Mikhail D. Lukin, ${ }^{3}$ Tao Shi, ${ }^{4}$ Yao Wang $\odot,{ }^{3}$ and Daniel Podolsky $\odot^{5, \dagger}$ \\ ${ }^{1}$ Max Planck Institute for the Structure and Dynamics of Matter, 22761 Hamburg, Germany \\ ${ }^{2}$ Max Planck Institute for Quantum Optics, 85748 Garching, Germany \\ ${ }^{3}$ Department of Physics, Harvard University, Cambridge, Massachusetts 02138, USA \\ ${ }^{4}$ CAS Key Laboratory of Theoretical Physics, Institute of Theoretical Physics, \\ Chinese Academy of Sciences, Beijing 100190, China \\ ${ }^{5}$ Physics Department, Technion, 32000 Haifa, Israel
}

(Received 29 August 2019; revised 25 September 2020; accepted 16 October 2020; published 17 March 2021)

\begin{abstract}
We propose a novel nonequilibrium phenomenon, through which a prompt quench from a metal to a transient superconducting state can induce large oscillations of the order parameter amplitude. We argue that this oscillating mode acts as a source of parametric amplification of the incident radiation. We report experimental results on optically driven $\mathrm{K}_{3} \mathrm{C}_{60}$ that are consistent with these predictions. The effect is found to disappear when the onset of the excitation becomes slower than the Higgs-mode period, consistent with the theory proposed here. These results open new possibilities for the use of collective modes in many-body systems to induce nonlinear optical effects.
\end{abstract}

DOI: $10.1103 /$ PhysRevX.11.011055

Subject Areas: Condensed Matter Physics, Strongly Correlated Materials, Superconductivity

\section{INTRODUCTION}

The quest for new functionalities in quantum materials has recently been extended to nonequilibrium states, which are interesting both because they exhibit new physical phenomena and because of their potential for high-speed device applications. Notable advances have been made in the creation of metastable phases [1-9] and in Floquet engineering under external periodic driving [10-12]. In the context of nonequilibrium superconductivity, examples have included the generation of transient superconductivity above the thermodynamic transition temperature $[6,8,9,13,14]$, the optical control of the interlayer phase in cuprates $[15,16]$, and the excitation of coherent Higgs-mode oscillations [17-20].

The Higgs mode is a fundamental collective excitation of systems with spontaneous symmetry breaking. It is a gapped excitation associated with oscillations of the amplitude of the order parameter. Examples of the Higgs mode in condensed matter are plentiful: It has been

\footnotetext{
*The first two authors have contributed equally.

Corresponding author.

podolsky@physics.technion.ac.il

Published by the American Physical Society under the terms of the Creative Commons Attribution 4.0 International license. Further distribution of this work must maintain attribution to the author(s) and the published article's title, journal citation, and DOI.
}

observed in the superconducting phase of $\mathrm{Nb}_{1-x} \mathrm{Ti}_{x} \mathrm{~N}$ [17-19], $\mathrm{Bi}_{2} \mathrm{Sr}_{2} \mathrm{CaCu}_{2} \mathrm{O}_{8+x}$ [20], $\mathrm{NbSe}_{2}$ [21-24], and in amorphous superconducting films [25]; in the dimerized antiferromagnet $\mathrm{TlCuCl}_{3}$ [26], in a variety of incommensurate charge density wave (CDW) systems [27-29], and in cold bosonic gases near the superfluid-to-Mott-insulator transition [30,31].

In a number of experiments reported recently, superconductivity is created nonadiabatically after a rapid change in microscopic interactions, as induced by the application of a terahertz or midinfrared (MIR) pump pulse $[6,8,9,13,14,32-35]$. Although the dynamics of this process are not yet fully understood, we posit that the "Mexican hat" effective potential for the superconducting order parameter is established promptly after optical excitation; see Fig. 1(a). The appearance of large Higgs oscillations is then a natural attribute of such photoinduced superconductivity if the quench is fast compared to the frequency of the Higgs mode $\omega_{H}$; see Fig. 1(b) (the order parameter dynamics following a sudden quantum quench has been studied theoretically in a number of related contexts; see, e.g., Refs. [36-39]).

We argue that in this situation, the coherent collective mode can act as a source of parametric amplification $[40,41]$ for oscillations of long-lived phase fluctuations, resulting in an enhanced reflectivity for a time-delayed probe pulse. This phenomenon can be qualitatively understood as a superconductor with an excited Higgs mode, which then places a parametric modulation on 

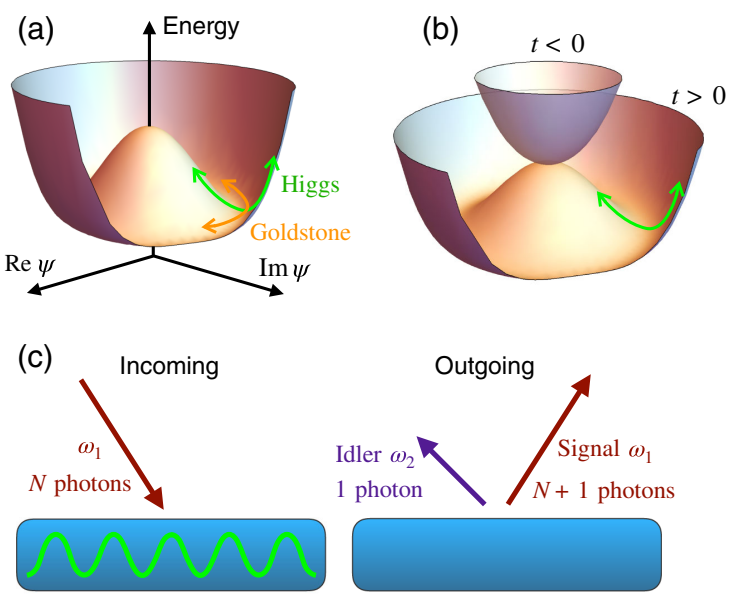

1 Higgs mode $\omega_{\mathrm{H}}$

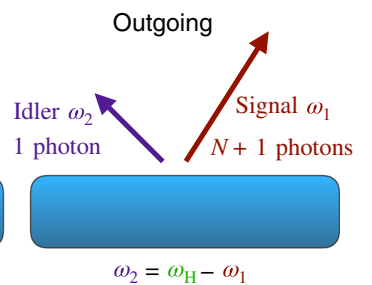

FIG. 1. (a) Schematic potential in a broken symmetry state. The collective excitations are Goldstone and Higgs modes. (b) Sudden change in system parameters at time $t=0$ leading to a rapid inversion in the potential, thus inducing Higgs oscillations. (c) Light amplification in a superconductor with an excited Higgs mode. Left panel: probe pulse containing $N$ photons of frequency $\omega_{1}$, incident on a superconductor with an excited Higgs mode (represented by a wave). Right panel: output consisting of $N+1$ photons of frequency $\omega_{1}$, plus a single photon of frequency $\omega_{2}$ traveling "backward," due to in-plane momentum conservation. When many Higgs excitations undergo stimulated decay, a large number of both $\omega_{1}$ and $\omega_{2}$ photons are produced.

low-frequency phase modes. We predict that the reflected beam would then feature amplification of intensity at the original frequency $\omega_{1}$, as well as the generation of an idler signal at the complementary frequency $\omega_{H}-\omega_{1}$ [see Fig. 1(c)]. We dub this phenomenon "Higgs amplification."

We also report experimental results on optically driven $\mathrm{K}_{3} \mathrm{C}_{60}$, which support these predictions. Using a pumpprobe technique, we illuminate our samples with a pump pulse at $41 \mathrm{THz}$ and a probe pulse spanning frequencies between 1 and $7 \mathrm{THz}$. Phase-resolved detection of the reflected signal allows us to reconstruct both the real and imaginary parts of the conductivity at the probe frequency. We find that when $\mathrm{K}_{3} \mathrm{C}_{60}$ is driven with pump pulses of suitable duration, the incident probe light is locally amplified near the surface for frequencies below $10 \mathrm{meV} / \hbar$. We analyze experimental results, taking into account the penetration depth mismatch between the pump and probe pulses. We observe an anomalous enhancement of the reflectance, which, in an homogeneously excited medium, would result in $6 \%$ amplification. For the penetration depth of the probe beam of $700 \mathrm{~nm}$, this corresponds to the amplification coefficient $\alpha \sim 10^{3} \mathrm{~cm}^{-1}$.

Our underlying theoretical considerations are presented in Secs. II and III. Experimental results are presented in Sec. IV, and a comparison of theory and experiment is given in Sec. V. Some implications of this work and the outlook for further developments are given in Sec. VI. Additional details of our analysis are given in three Appendixes.

\section{FROM HIGGS OSCILLATIONS TO ANOMALOUS REFLECTION}

The coupling between the Higgs mode and light can be understood by observing that in a general state with broken continuous symmetry, the Higgs mode can decay into a pair of Goldstone modes. For neutral superfluids, this process determines the lifetime of Higgs excitations [42]. In superconductors, the Goldstone mode describes phase fluctuations and therefore a charge current, which interacts directly with photons. Deep inside the material, these photons are gapped to the plasma frequency by the Anderson-Higgs mechanism, thus protecting the Higgs mode from decay into photons. On the other hand, near the surface, a Higgs excitation can decay into a pair of gapless vacuum photons whose frequencies add up to $\omega_{\mathrm{H}}$, which corresponds to an effective term in the Hamiltonian

$$
\mathcal{H}_{\text {Higgs } / \text { photons }} \sim \sum_{\omega_{1}+\omega_{2}=\omega_{\mathrm{H}}}\left(h a_{\omega_{1}}^{\dagger} a_{\omega_{2}}^{\dagger}+h^{\dagger} a_{\omega_{1}} a_{\omega_{2}}\right),
$$

where $h$ is a quantum field that annihilates a Higgs excitation and $a_{\omega}$ annihilates a vacuum photon of frequency $\omega$. Hence, the energy of the excited Higgs mode is converted into entangled photon pairs. In the absence of the probe pulse, all photon pairs are generated equally. However, the incoming probe enhances the generation of pairs in which one of the photons matches the incident photon frequency because of the bosonic stimulation factor.

One can understand the origin of the coupling in Eq. (1) from the following consideration. In superconductors, the diamagnetic coupling term between light and matter, $H_{\text {dia }}=\left(e^{2} \hbar^{2} / 2 m c^{2}\right) n_{\mathrm{s}} \mathbf{A}^{2}$, plays a key role, giving rise to the London equation for the current response to an electromagnetic field and to the Meissner effect. Here, $n_{\mathrm{s}}$ is the superfluid density, which, for a coherently excited Higgs mode, is expected to oscillate at the Higgs frequency, $n_{\mathrm{s}}=n_{s, 0}+\delta n \cos \left(\omega_{\mathrm{H}} t\right)$. In a quantized description of the electromagnetic field, the vector potential $\mathbf{A}$ is a linear combination of photon creation and annihilation operators, schematically of the form $\mathbf{A} \sim \sum_{\omega}\left(a_{\omega}+a_{\omega}^{\dagger}\right)$. This description implies that the diamagnetic term gives rise to the term (1), where $h$ is a quantum analog of the oscillating part of the superfluid density $\delta n$. Interactions of this type are known to give rise to stimulated emission and parametric down conversion of light [43].

These considerations give rise to the following mechanism of Higgs amplification. Consider an incident probe pulse composed of $N$ photons at frequency $\omega_{1}<\omega_{H}$ as it is reflected from a superconductor. The term $a_{\omega_{1}}^{\dagger} a_{\omega_{2}}^{\dagger}$ creates a pair of photons, leading to a state with $N+1$ photons at $\omega_{1}$ 
and one $\omega_{2}=\omega_{H}-\omega_{1}$ photon; see Fig. 1(c). The amplitude of this process is enhanced by a Bose factor of $\sqrt{N+1}$. As long as the Higgs mode remains excited, this process leads to amplification of $\omega_{1}$ photons and generation of $\omega_{2}$ photons, until the Higgs mode is depleted. The net effect is outgoing light with two frequencies, $\omega_{1}$ and $\omega_{2}$, related by $\omega_{1}+\omega_{2}=\omega_{\mathrm{H}}$, and the outgoing light at $\omega_{1}$ having a higher intensity than the incoming signal.

In typical superconductors, the Higgs frequency is smaller than the plasma frequency. Then, incident light with $\omega_{1}<\omega_{H}$ does not penetrate deeply into the material, and Higgs amplification is a surface effect. In particular, because of the evanescent nature of the waves inside the material, there are no phase-matching conditions in this process. Thus, there is not a discrete set of frequencies at which Higgs amplification is resonantly enhanced, and hence the level of amplification is expected to depend smoothly on the probe frequency $\omega_{1}$. The frequency scale for the Higgs mode is comparable to the superconducting gap. Hence, for many superconductors, Higgs amplification is expected to occur in the terahertz, a frequency range that is of great current interest for fundamental science and technology applications $[44,45]$. The next section presents a calculation of Higgs amplification based on a semiclassical description of photons using Maxwell's equations. Our discussion is agnostic about the specific microscopic mechanism underlying light-induced superconductivity and, assuming that Higgs oscillations have been coherently excited, directly studies their effect on optical properties.

\section{OPTICAL PROPERTIES OF A SUPERCONDUCTOR WITH AN EXCITED HIGGS MODE}

The optical properties of a superconductor with an excited Higgs mode are understood as follows. Consider Maxwell's equations combined with the relation between the electrical current in the material and electric field:

$$
\begin{gathered}
\nabla \times \mathbf{B}-\frac{\epsilon}{c^{2}} \frac{\partial \mathbf{E}}{\partial t}=\mu_{0} \mathbf{j}, \\
\nabla \times \mathbf{E}+\frac{\partial \mathbf{B}}{\partial t}=0 .
\end{gathered}
$$

Here, $\epsilon$ is the dielectric constant arising from bound charges, and $\mathbf{j}$ is the free current density. For $z>0$, outside the superconductor, $\epsilon=\epsilon_{\text {out }}$ (in vacuum, $\epsilon_{\text {out }}=1$, but we allow for interfaces with other media), and we assume the current $\mathbf{j}$ vanishes; for $z<0, \epsilon=\epsilon_{\mathrm{s}}$, and the current satisfies the London equation,

$$
\mathbf{j}=\Lambda(t) \mathbf{v}_{\mathrm{s}} .
$$

Here, $\mathbf{v}_{\mathrm{s}}=(\hbar / 2 e) \nabla \theta-\mathbf{A}$, where $\theta$ is the superconducting phase and $\mathbf{A}$ is the vector potential. Note that $\partial_{t} \mathbf{v}_{\mathrm{s}}=\mathbf{E}$.
In an equilibrium superconductor, $\Lambda(t)$ reduces to the static value $\Lambda_{\mathrm{s}}=\left(e^{2} n_{\mathrm{s}} / m\right)$, where $n_{\mathrm{s}}$ is the superfluid density. More generally, in a time-modulated superconductor, Eq. (4) accurately describes the total current induced by a vector potential $\mathbf{A}$ in the limit where $\mathbf{A}$ and $\Lambda$ vary slowly compared to any microscopic frequencies such as the superconducting energy gap and the scattering rate $1 / \tau$ for electrons in the normal state. We use it here under the assumption that it is at least a good starting approximation for the frequencies of interest to us. Equation (4) will later be supplemented by a more general relation in which the effects of dissipation are taken into account. A more general description of the electromagnetic response of a Higgsmodulated superconductor is provided in Appendix A.

Generally, the value of $\Lambda$ will depend on the value of the superconducting energy gap. (Although the special case of an ideal clean superconductor at $T=0$ is an exception to this rule, we expect that the gap dependence will be manifest in the systems of interest to us because of the polaronic effects and coupling to impurities [46].) Since the Higgs mode represents a modulation in the energy gap, we expect it to induce a similar modulation in $\Lambda$. Consequently, we may write

$$
\Lambda(t)=\Lambda_{\mathrm{s}}+\Lambda_{\mathrm{m}} e^{-i \omega_{\mathrm{H}} t}+\Lambda_{\mathrm{m}}^{*} e^{i \omega_{\mathrm{H}} t},
$$

where $\Lambda_{\mathrm{m}}$ describes the amplitude of the modulation and $\omega_{\mathrm{H}}$ is the Higgs-mode frequency. For a BCS superconductor, $\omega_{\mathrm{H}}=2 \Delta$, where $\Delta$ is the quasiparticle gap [22].

Different frequencies mix because of the time dependence in $\Lambda$ : Incident light with frequency $\omega_{1}$ will produce outgoing light with frequencies $\omega_{1}$ and $\omega_{2}=\omega_{\mathrm{H}}-\omega_{1}$; see Fig. 1(c). Mixing of the Higgs modulation and the signal beam is also expected to induce light at the frequency $\omega_{3}=\omega_{1}+\omega_{H}$. We note, however, that the $\omega_{3}$ frequency lies well above the quasiparticle threshold $2 \Delta$, and we expect that this channel of mode mixing will be suppressed relative to the channel at $\omega_{2}$. This case can be understood by observing that at such high frequencies, the current should be carried primarily by quasiparticles, rather than supercurrents, and one might expect the quasiparticle current to be less sensitive than the supercurrent to modulations of the superconducting amplitude. In the remainder of this paper, for the sake of simplicity, we assume that the excitation of the $\omega_{3}$ mode is negligible, and we omit it entirely from our considerations. Our results should be qualitatively applicable, however, as long as the excitation of the $\omega_{3}$ mode is substantially weaker than that of the $\omega_{2}$ mode.

Prior to solving the reflection problem, it is useful to first consider the evanescent wave solutions inside the superconductor. To simplify our discussion, we assume here that $\Lambda_{m}$ is spatially uniform inside the superconductor. Nonuniformities in $\Lambda_{m}$ due to the short penetration depth of the exciting radiation will be taken into account in the 
processing of the experimental data, as detailed in Appendix B. For the case of uniform $\Lambda_{m}$, the evanescent solutions are characterized by the spacetime dependence ( $\mathbf{V}$ stands for $\mathbf{j}, \mathbf{v}_{\mathrm{s}}, \mathbf{E}$, or $\mathbf{B}$ ),

$$
\mathbf{V}=\left(\mathbf{V}_{1} e^{-i \omega_{1} t}+\mathbf{V}_{2}^{*} e^{i \omega_{2} t}\right) e^{\kappa z}+\text { c.c. }
$$

Note that the same spatial dependence appears for $\omega_{1}$ and $-\omega_{2}$ terms since they mix with each other homogeneously in space. This case should be contrasted with the static case, where each frequency mode decays with its own $\kappa$. Substituting Eq. (6) into Eq. (4) and collecting terms with frequencies $\omega_{1}$ and $-\omega_{2}$ yields

$$
\begin{aligned}
& \mathbf{j}_{1}=\Lambda_{\mathrm{s}} \mathbf{v}_{\mathrm{s}, 1}+\Lambda_{\mathrm{m}} \mathbf{v}_{\mathrm{s}, 2}^{*}=\Lambda_{\mathrm{s}} \frac{\mathbf{E}_{1}}{-i \omega_{1}}+\Lambda_{\mathrm{m}} \frac{\mathbf{E}_{2}^{*}}{i \omega_{2}}, \\
& \mathbf{j}_{2}^{*}=\Lambda_{\mathrm{s}} \mathbf{v}_{\mathrm{s}, 2}^{*}+\Lambda_{\mathrm{m}}^{*} \mathbf{v}_{\mathrm{s}, 1}=\Lambda_{\mathrm{s}} \frac{\mathbf{E}_{2}^{*}}{i \omega_{2}}+\Lambda_{\mathrm{m}}^{*} \frac{\mathbf{E}_{1}}{-i \omega_{1}} .
\end{aligned}
$$

For linearly polarized light, $\mathbf{E}_{\nu}=E_{\nu} \hat{e}_{x}, \mathbf{B}_{\nu}=B_{\nu} \hat{e}_{y}$, and $\mathbf{j}_{\nu}=j_{\nu} \hat{e}_{x}$, where $\nu \in\{1,2\}$. Then, Eq. (3) yields $B_{\nu}=\left(\kappa / i \omega_{\nu}\right) E_{\nu}$, and Eq. (2) becomes

$$
\left(\begin{array}{cc}
\kappa^{2} c^{2}+\omega_{1}^{2} \epsilon_{1} & \Upsilon \omega_{1} / \omega_{2} \\
\Upsilon^{*} \omega_{2} / \omega_{1} & \kappa^{2} c^{2}+\omega_{2}^{2} \epsilon_{\overline{2}}
\end{array}\right)\left(\begin{array}{c}
E_{1} \\
E_{2}^{*}
\end{array}\right)=0 .
$$

Here, $\Upsilon \equiv \Lambda_{\mathrm{m}} / \varepsilon_{0}, \epsilon_{1} \equiv \epsilon\left(\omega_{1}\right)$, and $\epsilon_{\overline{2}} \equiv \epsilon\left(-\omega_{2}\right)$, where $\epsilon(\omega)=\epsilon_{\mathrm{s}}-\Lambda_{\mathrm{s}} /\left(\varepsilon_{0} \omega\left(\omega+i 0^{+}\right)\right)$is the dielectric function of the superconductor. [Note that, by changing the form of $\epsilon(\omega)$, one can generalize the discussion, e.g., to introduce dissipation.] The allowed values of $\kappa$ are obtained by requiring the determinant of the above matrix to vanish,

$\kappa_{ \pm}^{2} c^{2}=-\frac{\omega_{1}^{2} \epsilon_{1}+\omega_{2}^{2} \epsilon_{\overline{2}}}{2} \pm \sqrt{\frac{\left(\omega_{1}^{2} \epsilon_{1}-\omega_{2}^{2} \epsilon_{\overline{2}}\right)^{2}}{4}+|\Upsilon|^{2}}$,

and the fields inside the superconductor satisfy

$$
\begin{gathered}
\frac{E_{1, \pm}^{\mathrm{t}}}{B_{1, \pm}^{\mathrm{t}}}=\frac{i \omega_{1}}{\kappa_{ \pm}} \equiv \eta_{ \pm}, \\
\frac{E_{2, \pm}^{\mathrm{t} *}}{B_{1, \pm}^{\mathrm{t}}}=\frac{-i \omega_{2}\left(\kappa_{ \pm}^{2} c^{2}+\omega_{1}^{2} \epsilon_{1}\right)}{\Upsilon \kappa_{ \pm}} \equiv \phi_{ \pm}, \\
\frac{B_{2, \pm}^{\mathrm{t*}}}{B_{1, \pm}^{\mathrm{t}}}=\frac{\left(\kappa_{ \pm}^{2} c^{2}+\omega_{1}^{2} \epsilon_{1}\right)}{\Upsilon} \equiv \gamma_{ \pm},
\end{gathered}
$$

where the superscript $t$ indicates that these act as transmitted fields in the reflection problem.

Next, we consider a normally incident signal beam of frequency $\omega_{1}$, and reflected beams at frequencies $\omega_{1}$ and $\omega_{2}$, with

$$
\begin{aligned}
& \mathbf{E}_{1}^{\mathrm{i}}, \mathbf{B}_{1}^{\mathrm{i}} \propto e^{-i \omega_{1}\left(t+z \sqrt{\epsilon_{\text {out }}} / c\right)}, \\
& \mathbf{E}_{\nu}^{\mathrm{r}}, \mathbf{B}_{\nu}^{\mathrm{r}} \propto e^{-i \omega_{\nu}\left(t-z \sqrt{\epsilon_{\text {out }}} / c\right)},
\end{aligned}
$$

where $\nu \in\{1,2\}$. The fields are linearly polarized as before, $\mathbf{E}_{\nu}=E_{\nu} \hat{e}_{x}, \mathbf{B}_{\nu}=B_{\nu} \hat{e}_{y}$, and Maxwell's equations constrain them to satisfy $E_{1}^{\mathrm{i}}=-\left(c / \sqrt{\epsilon_{\text {out }}}\right) B_{1}^{\mathrm{i}}$ and $E_{\nu}^{\mathrm{r}}=$ $\left(c / \sqrt{\epsilon_{\text {out }}}\right) B_{\nu}^{\mathrm{r}}$. Within the superconductor, the transmitted fields are superpositions of two evanescent waves of the form (6) with $\kappa=\kappa_{ \pm}$, whose amplitudes are fixed by boundary conditions imposed independently on each frequency:

$$
\begin{gathered}
B_{1}^{\mathrm{i}}+B_{1}^{\mathrm{r}}=B_{1,+}^{\mathrm{t}}+B_{1,-}^{\mathrm{t}}, \\
E_{1}^{\mathrm{i}}+E_{1}^{\mathrm{r}}=E_{1,+}^{\mathrm{t}}+E_{1,-}^{\mathrm{t}}, \\
B_{2}^{\mathrm{r} *}=B_{2,+}^{\mathrm{t} *}+B_{2,-}^{\mathrm{t} *}, \\
E_{2}^{\mathrm{r} *}=E_{2,+}^{\mathrm{t} *}+E_{2,-}^{\mathrm{t} *},
\end{gathered}
$$

Combining these with Eqs. (11)-(13) yields

$$
\begin{gathered}
r \equiv \frac{B_{1}^{\mathrm{r}}}{B_{1}^{\mathrm{i}}}=\frac{(1+\zeta)+\sqrt{\epsilon_{\mathrm{out}}}\left(\eta_{+}+\zeta \eta_{-}\right)}{(1+\zeta)-\sqrt{\epsilon_{\mathrm{out}}}\left(\eta_{+}+\zeta \eta_{-}\right)}, \\
r_{12} \equiv \frac{B_{2}^{\mathrm{r} *}}{B_{1}^{\mathrm{i}}}=\frac{2\left(\gamma_{+}+\zeta \gamma_{-}\right)}{(1+\zeta)-\sqrt{\epsilon_{\mathrm{out}}}\left(\eta_{+}+\zeta \eta_{-}\right)},
\end{gathered}
$$

where

$$
\zeta=-\frac{\gamma_{+}-\sqrt{\epsilon_{\mathrm{out}}} \phi_{+}}{\gamma_{-}-\sqrt{\epsilon_{\mathrm{out}}} \phi_{-}}
$$

Equations (20) and (21) are the main theoretical results of this paper: $r$ is the reflection amplitude of the signal beam, whereas $r_{12}$ is the amplitude of the emitted idler mode at the downconverted frequency $\omega_{2}=\omega_{\mathrm{H}}-\omega_{1}$, normalized by the amplitude of the incident signal beam.

Figure 2 shows $R=|r|^{2}$ and $R_{12}=\left|r_{12}\right|^{2}$ as a function of $\omega_{1}$, in the case $\omega_{\mathrm{H}}<\omega_{\mathrm{ps}}$, where $\omega_{\mathrm{ps}}=\sqrt{\Lambda_{\mathrm{s}} /\left(\varepsilon_{0} \epsilon_{\mathrm{s}}\right)}$ is the superconducting plasma frequency of the material. Note that, in the absence of dissipation, there is amplification $R>1$ over the entire range $0<\omega_{1}<\omega_{\mathrm{H}}$, and the maximum amplification occurs at $\omega_{1}=\omega_{\mathrm{H}} / 2$. One can study the effect of dissipation by writing $\epsilon(\omega)=$ $\epsilon_{\mathrm{s}}-\sigma(\omega) /\left(i \varepsilon_{0} \omega\right)$ in Eqs. (20) and (21) and by adding a real part to the conductivity function $\sigma(\omega)$. This process suppresses the reflectivity and also reduces the frequency $\omega_{1}$ at which the maximum occurs, as shown in Fig. 2(a).

Note that the net amplification in Fig. 2 is small, of the order of a few percent. In order to understand this, we focus on $\omega_{1}=\omega_{2}=\omega_{\mathrm{H}} / 2$, where the maximum is obtained, with the value 

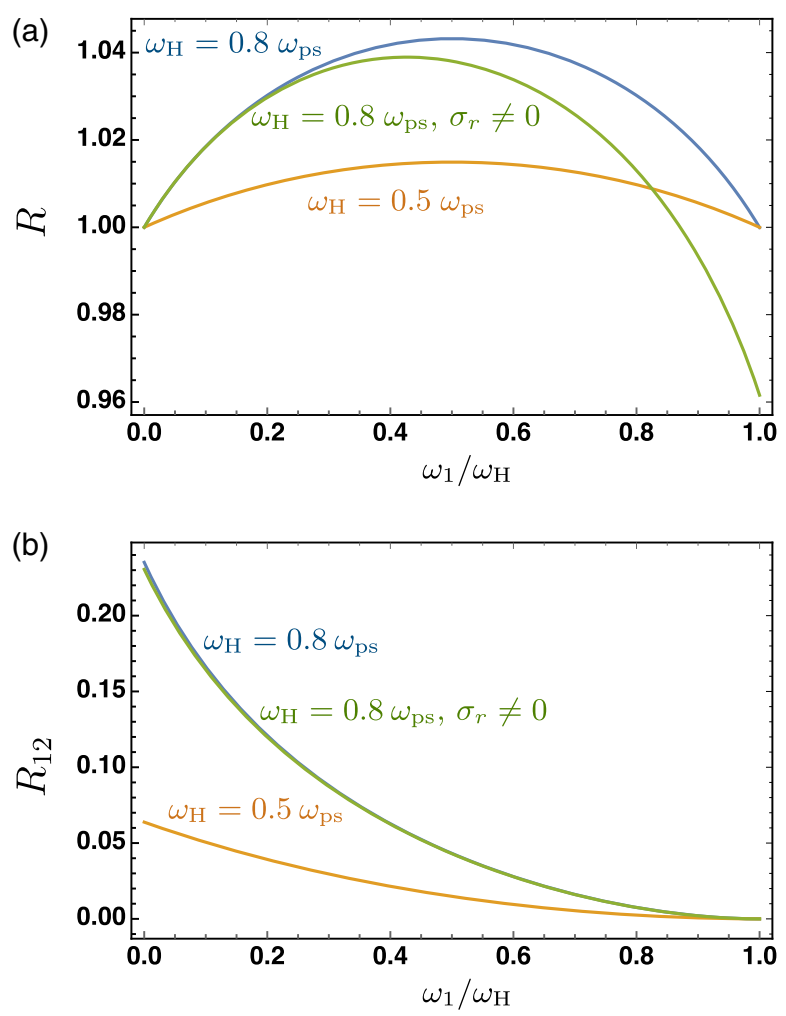

FIG. 2. (a) Reflection coefficient $R$ and (b) downconversion intensity $R_{12}$ as a function of the signal frequency $\omega_{1}$. We assume $\epsilon_{\mathrm{s}}=5, \epsilon_{\text {out }}=5.62$ (corresponding to diamond), and $\Lambda_{\mathrm{m}}=0.4 \Lambda_{\mathrm{s}}$. Two different values of the Higgs frequency were taken, $\omega_{\mathrm{H}}=$ $0.5 \omega_{\mathrm{ps}}$ and $\omega_{\mathrm{H}}=0.8 \omega_{\mathrm{ps}}$, where $\omega_{\mathrm{ps}}=\sqrt{\Lambda_{\mathrm{s}} /\left(\varepsilon_{0} \epsilon_{\mathrm{s}}\right)}$ is the superconducting plasma frequency. The green curves, corresponding to $\omega_{\mathrm{H}}=0.8 \omega_{\mathrm{ps}}$, illustrate the effects of dissipation, taken into account by assuming $\epsilon(\omega)=\epsilon_{\mathrm{s}}-\sigma(\omega) /\left(i \varepsilon_{0} \omega\right)$ with $\sigma(\omega)=$ $\sigma_{\mathrm{r}}+i \Lambda_{\mathrm{s}} /\left(\omega+i 0^{+}\right)$, where $\sigma_{\mathrm{r}}$ is a real constant; the blue and orange curves include no dissipation, $\sigma_{\mathrm{r}}=0$. Results are for the model in which $\Lambda_{m}$ is uniform in space.

$$
R_{\max }=1+\frac{\epsilon_{\text {out }} \omega_{\mathrm{H}}^{2}\left(D_{+}-D_{-}\right)^{2}}{\left[\epsilon_{\text {out }} \omega_{\mathrm{H}}^{2}+D_{+} D_{-}\right]^{2}},
$$

where $D_{ \pm}=\sqrt{4\left(\Lambda_{\mathrm{s}} \pm\left|\Lambda_{\mathrm{m}}\right|\right) / \varepsilon_{0}-\epsilon_{\mathrm{s}} \omega_{\mathrm{H}}^{2}}$. For $\omega_{\mathrm{H}}^{2} \ll \Lambda_{\mathrm{s}} / \varepsilon_{0}$ and $\Lambda_{\mathrm{m}} \ll \Lambda_{\mathrm{s}}$, Eq. (23) becomes

$$
R_{\max }=1+\frac{\left|\Lambda_{\mathrm{m}}\right|^{2}}{\Lambda_{\mathrm{s}}^{2}} \frac{\epsilon_{\mathrm{out}} \omega_{\mathrm{H}}^{2}}{4 \epsilon_{\mathrm{s}} \omega_{\mathrm{ps}}^{2}} .
$$

The factor $\left|\Lambda_{\mathrm{m}}\right|^{2} / \Lambda_{\mathrm{s}}^{2}$ expresses the fact that the amplification is proportional to the intensity of the modulation. The factor $\epsilon_{\text {out }} \omega_{\mathrm{H}}^{2} /\left(4 \epsilon_{\mathrm{s}} \omega_{\mathrm{ps}}^{2}\right)$ is the square of the London penetration depth divided by the wavelength of the incident light; it expresses the fact that amplification is weak if the light cannot probe deeply into the superconductor. This observation suggests that, in order to enhance the amplification, one could consider instances in which the light can probe a larger region of the superconductor prior to being reflected. Two possible approaches to achieve this result come to mind: first, by using incoming light with a shallow incidence angle to the sample, thus introducing geometrical factors that enhance the effect; second, by studying systems in which the Higgs frequency exceeds the plasma frequency. These possibilities will be discussed elsewhere [47]. Note that, in a realistic superconductor, the penetration of light is controlled by the total plasma frequency $\omega_{\mathrm{p}}$, which receives contributions from all charge carriers, not just the superconducting ones. It is this total plasma frequency that presumably controls the strength of the Higgs amplification in Eq. (24).

In the next section, we present experimental evidence for Higgs amplification in the superconductor $\mathrm{K}_{3} \mathrm{C}_{60}$. This material is a natural candidate system for Higgs amplification. In this compound, the low density of electrons (three per $\mathrm{C}_{60}$ molecule) and the weak hopping between $\mathrm{C}_{60}$ molecules conspire to yield an anomalously small plasma frequency $\omega_{\mathrm{p}}=72 \mathrm{meV} / \hbar$. Optical excitation at midinfrared wavelengths has been shown to transform the high-temperature $\left(T \gg T_{c}\right)$ metallic phase of $\mathrm{K}_{3} \mathrm{C}_{60}$ into a transient nonequilibrium state with the same optical properties as the low-temperature superconductor $\left(T<T_{c}\right)$. The transient state, which is thought to be a photoinduced nonequilibrium superconductor, displays a saturated reflectivity $(R=1)$, a gap in the real part of the optical conductivity $\sigma_{1}$, and a divergent low-frequency imaginary conductivity $\sigma_{2}$ [8].

It is difficult to make an a priori estimation of $\omega_{\mathrm{H}}$. One possible approach would be to choose $\omega_{\mathrm{H}}=2 \Delta / \hbar$, as expected of a weakly coupled BCS superconductor. However, beyond the questions regarding the applicability of BCS theory to the photoinduced state of $\mathrm{K}_{3} \mathrm{C}_{60}$, a direct experimental determination of $\Delta$ is not possible since the superconducting gap is hidden behind a broad midinfrared absorption peak in the optical conductivity, as discussed below. In what follows, we find that the analysis of the measured reflection based on the predictions of Higgs amplification implies a Higgs frequency $\omega_{\mathrm{H}} \approx 24 \mathrm{meV} / \hbar$. Hence, $\mathrm{K}_{3} \mathrm{C}_{60}$ combines a relatively large value of $\omega_{\mathrm{H}} / \omega_{\mathrm{p}}$, as is necessary to enhance the reflectance in Eq. (24), together with the possibility for rapid quenches into the superconducting state by pump pulses, as needed to induce large Higgs oscillations. In particular, by modifying the type of quench protocol used, one may control the amplitude of Higgs oscillations.

In order to compare to experiments in photoinduced superconductors, we must relate to two considerations that are not taken into account in our model. The first one concerns the Higgs decay mechanism. In a realistic system, the Higgs oscillations are damped by decay into quasiparticles in a process that is kinematically marginal, leading to a slow, power-law damping [48-52], which is eventually expected to be superseded by a faster decay, since the lightinduced superconductivity itself is short-lived. In our 
analysis, the decay of the Higgs mode can be taken into account by replacing the monochromatic oscillations in the superfluid density by an integral:

$$
\Lambda(t)=\Lambda_{s}+\int d \omega \Lambda(\omega) e^{i \omega t},
$$

where $\Lambda_{s}$ is the static superfluid density. We regain the monochromatic case for

$$
\Lambda(\omega)=\Lambda_{m} \delta\left(\omega-\omega_{H}\right)+\Lambda_{m}^{*} \delta\left(\omega+\omega_{H}\right),
$$

but, more generally, Higgs decay requires broadening of these delta functions into complex functions of nonzero width.

Each frequency $\omega$, when considered in isolation, gives rise to amplification in proportion to $|\Lambda(\omega)|^{2}$; see, e.g., Eq. (24). When considered in conjunction, the total amplification is then the integral over the amplification due to each independent frequency, weighted by $|\Lambda(\omega)|^{2}$. This result is correct to leading order in the Higgs amplitude and may receive corrections of order $|\Lambda|^{4}$. There are two different ways to see this. One is from a quantum point of view, starting from Eq. (1). Signal amplification arising from different Higgs frequency components gives rise to idlers of different frequencies. To the extent that these idlers are classical beams, these processes are not quantum coherent, and their amplitudes must add in quadrature. Another, more general consideration, is that to leading order in the Higgs oscillations, signal amplification cannot depend on the relative phase of different Fourier components $\Lambda(\omega)$. This result implies that the predicted signal amplification is relatively robust to broadening arising from a finite Higgs lifetime since the amplification factors due to independent frequencies simply add up. As alluded to above, the idler beam is much less robust, as it will get broadened in frequency. Hence, an independent observation of the idler spectrum would not only give information about the Higgs frequency but also about its decay.

The second consideration relates to the lack of global phase coherence: When quenching from the normal state into a superconducting phase, superconductivity nucleates locally in different regions of the sample (whose characteristic size is given by the coherence length). Then, the spontaneously broken phase is expected to vary spatially across different regions for the entire duration of the experiment because the coarsening dynamics for the phase is very slow, preventing the formation of global phase coherence over the lifetime of the photoinduced state. In contrast to the phase, in the clean-sample limit, the amplitude of the superconducting order parameter is expected to be relatively homogeneous across the sample (but oscillating in time because of excitation of the Higgs mode). Higgs amplification is caused by the oscillations in amplitude of the superconducting order parameter, which are not strongly affected by the phase inhomogeneity. In principle, one may worry about the interaction of light with the supercurrents associated (through the Josephson relation) with gradients in the superconducting phase. However, to the extent that the coarsening dynamics are slow, these supercurrents vary only slowly in time, and they do not couple to electromagnetic radiation at the frequencies probed in the experiment. Hence, we expect the mechanism of Higgs amplification to occur, despite the lack of global phase coherence.

\section{EXPERIMENTAL RESULTS}

We follow the same protocol described in Refs. $[8,9]$ to photoinduce the transient optical properties of a nonequilibrium superconductor in $\mathrm{K}_{3} \mathrm{C}_{60}$ but using shorter and more intense pulses. To test the hypotheses discussed in the theory section above, we additionally analyze conditions in which the quench is made slower with respect to the Higgs-mode frequency of the photoinduced state. The pump-pulse FWHM duration $\tau$ is tuned to different values between $100 \mathrm{fs}$ and $1.8 \mathrm{ps}$. This range of pulse durations is interesting because it crosses a characteristic timescale $\tau^{*}=2 \pi / \omega_{\mathrm{H}}=h /(24 \mathrm{meV}) \sim 172 \mathrm{fs}$, which corresponds to the period of the amplitude (Higgs) mode in the photoinduced superconductor. Note that in our experimental geometry, the idler mode is not detected. In future studies, this mode could provide a measurement of the Higgs frequency and would allow for distinguishing Higgs amplification from other types of parametric amplification [53,54].

In the excitation regime explored in this experiment, when the pump pulse is significantly longer than $\tau^{*}$, the transient state displays a reflection coefficient at the surface that is saturated at $R=1$ for all frequencies below about $10 \mathrm{meV}$. By reconstructing the complex optical conductivity with the same procedure used previously [9], we extract a gapped real optical conductivity $\sigma_{1}$ at all frequencies $\omega<10 \mathrm{meV}$ and a divergent low-frequency imaginary conductivity $\sigma_{2}$, indicative of a light-induced superconducting state. Our experiment reveals that as the pulse duration is made progressively shorter than $\tau^{*}$, the transient state acquires a reflection coefficient that is larger than $R=1$ immediately after the pump, indicative of optical amplification through the nonadiabatic creation of a superconducting state. The real part of the optical conductivity $\sigma_{1}$ is negative at all frequencies, $\omega<10 \mathrm{meV}$, while its imaginary part $\sigma_{2}$ remains divergent. Conceptually, the observed dependence on the pulse duration can be understood from the following consideration. Although the underlying mechanism of photoinduced superconductivity is still the subject of debate, we assume, as a first approximation, that the "effective" final-state Hamiltonian experienced by the low-energy electrons depends only on the total pulse energy and not its duration (later, we will show evidence 
that the shorter pulses actually drive the superconductivity more strongly and induce a slightly larger superfluid density, although this is a weak effect). Furthermore, we assume that the superconducting state lasts longer than the probe sequence. Hence, qualitatively, we assume that by controlling the pump-pulse duration, we preserve the final effective Hamiltonian for electrons but change the rate at which the microscopic parameters are modified. In the case of a superconductor, we expect that the Higgs-amplitude mode gets strongly excited when the interaction strength is modified on a timescale shorter than $\tau^{*}=2 \pi / \omega_{\mathrm{H}}$.

$\mathrm{K}_{3} \mathrm{C}_{60}$ polycrystalline powders were excited at normal incidence with $170-\mathrm{meV}$ linearly polarized, midinfrared pulses. Their duration was tuned from 100 fs to 1.8 ps by chirping them through linear propagation in transparent and highly dispersive $\mathrm{CaF}_{2}$ rods. For all pulse durations, the pulse energy and the number of incident photons were maintained at a constant level. The transient, lowfrequency, optical properties of photoexcited $\mathrm{K}_{3} \mathrm{C}_{60}$ were retrieved as a function of pump-probe delay using transient, terahertz, time-domain spectroscopy using $\mathrm{THz}$ pulses with a bandwidth that ranged from 1 to $7 \mathrm{THz}$. These probe pulses were made to strike the sample at near-normal incidence, with a $7^{\circ}$ incidence angle (see Fig. 3), and they were $p$-polarized, that is, with the electric field perpendicular to that of the MIR pump pulses. The measurement of the electric field reflected from the sample yielded a phase-resolved measurement of the reflection coefficient and, through it, the complex optical properties. The penetration depth of the midinfrared pump (200 nm) was shorter than that of the THz probe $(600-900 \mathrm{~nm})$. To account for this difference, the data were analyzed as discussed in Appendix B in order to obtain the reflectivity corresponding to an effective semi-infinite and homogeneously pumped medium.

Figures 4(a) and 4(b) compare the transient optical properties of $\mathrm{K}_{3} \mathrm{C}_{60}$ upon photoexcitation with intense midinfrared pulses of 1.8-ps and 100-fs duration,

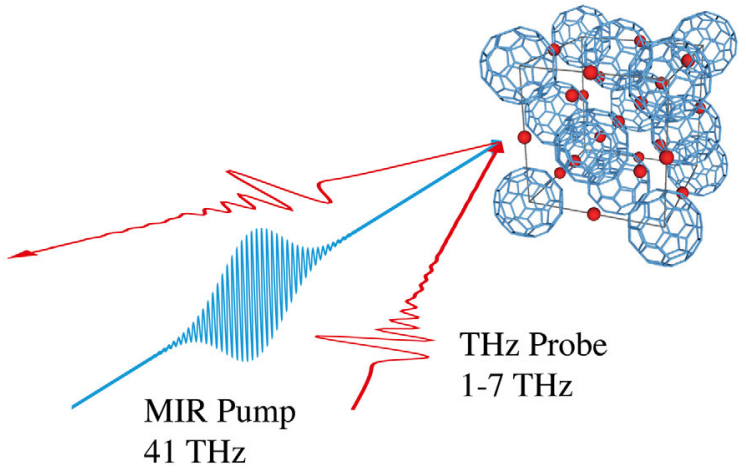

FIG. 3. Sketch of the experimental geometry. $\mathrm{K}_{3} \mathrm{C}_{60}$ is excited with vertically polarized midinfrared pulses. The $\mathrm{THz}$ probe pulses are polarized in the horizontal plane. respectively. The red curves report the optical properties of the equilibrium metallic state, while the light-blue dots represent those of the transient state, measured at the peak of the response. For longer excitation pulses [1.8 ps, Fig. 4(a)], the transient optical properties resemble those of the equilibrium superconductor with a reflectivity saturated at $R=1$, a gapped real conductivity $\left(\sigma_{1} \approx 0\right)$, and a divergent imaginary conductivity for all frequencies below about $10 \mathrm{meV}$. For $\tau=100 \mathrm{fs}$ [Fig. 4(b)], we find that below about $10 \mathrm{meV}$, the reflectivity becomes larger than $R=1$, reaching an average value in the gapped region of about 1.04, with a maximum of about 1.06. The extracted real part of the optical conductivity is correspondingly negative, indicative of negative dissipation. These two observations suggest amplification of the incoming low-frequency $\mathrm{THz}$ probe light. Importantly, the imaginary part of the optical conductivity maintains a $1 / \omega$ behavior below about $10 \mathrm{meV}$, indicating the superconducting nature of the transient state.

The evolution of the optical properties for pump pulses either shorter or longer than $\tau^{*}$ can be captured by the average value of the reflectivity in the 5-8- $\mathrm{meV}$ frequency range and the superfluid density extracted by a $1 / \omega$ fit to the imaginary part of the optical conductivity at low frequencies. Figures 5(a) and 5(b) show the evolution of these two quantities as a function of the duration of the excitation pulse [Fig. 5(b)]. The average reflectivity in the gapped region decreases from about 1.04 to about 1 , as the pump-pulse duration varies from 100 fs to $1.8 \mathrm{ps}$. The blue-shaded area in the top panel highlights the regime where light amplification is observed. At the same time, the superfluid density of the photoinduced superconductor does not appear to strongly depend on the pulse duration of the excitation pulse. In Appendix $\mathrm{C}$, we show that the observed amplification cannot be explained by heating of the sample upon photoexcitation.

It is also interesting to explore the dependence of the light amplification on the sample temperature. Figure 6 shows the temperature dependence of the frequency-averaged reflectivity and of the superfluid density, both measured at a fixed pump-pulse duration of $100 \mathrm{fs}$. Even at the highest measured temperature, $300 \mathrm{~K}$, the system behaves as a superconductor with a superfluid density comparable to that of the equilibrium superconductor at low temperatures. However, the frequency-averaged reflectivity is approximately 1 ; i.e., there is no amplification at $T=300 \mathrm{~K}$, which may indicate that the Higgs mode becomes overdamped at high temperatures, reminiscent of what occurs in equilibrium as the critical temperature of the superconductor is approached, when the order parameter dynamics become overdamped. As the temperature is lowered, we find that the data are qualitatively similar at the two intermediate temperatures, $100 \mathrm{~K}$ and $200 \mathrm{~K}$, indicating that the phenomenon of Higgs amplification is robust over a wide temperature window. 
(a) Slow quench (1.8 ps)
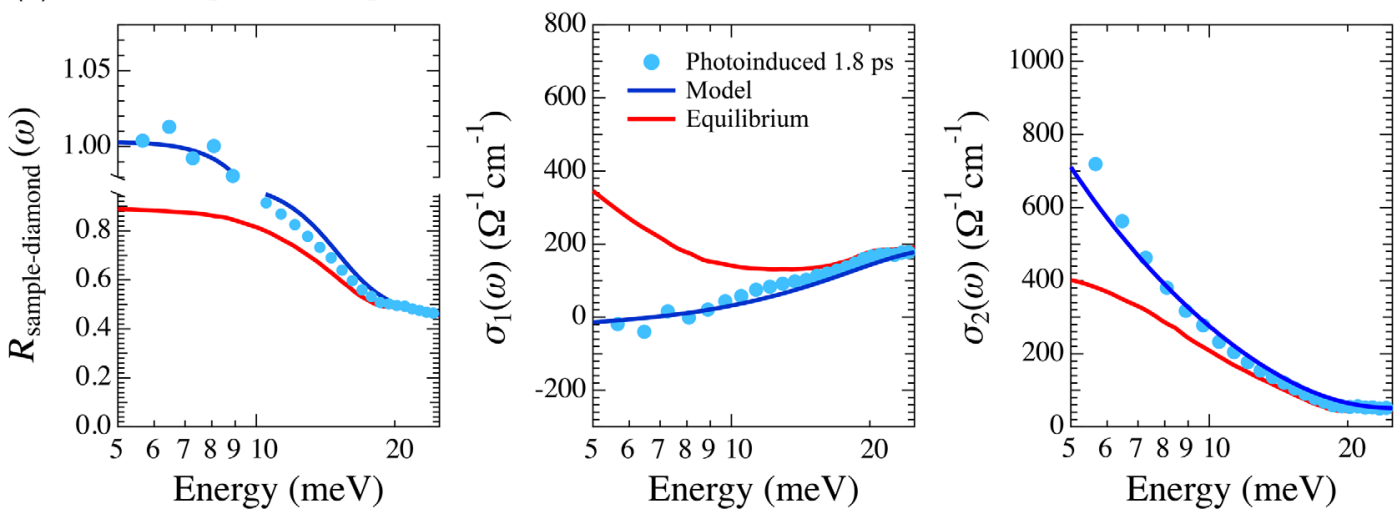

(b) Prompt quench $(0.1 \mathrm{ps})$
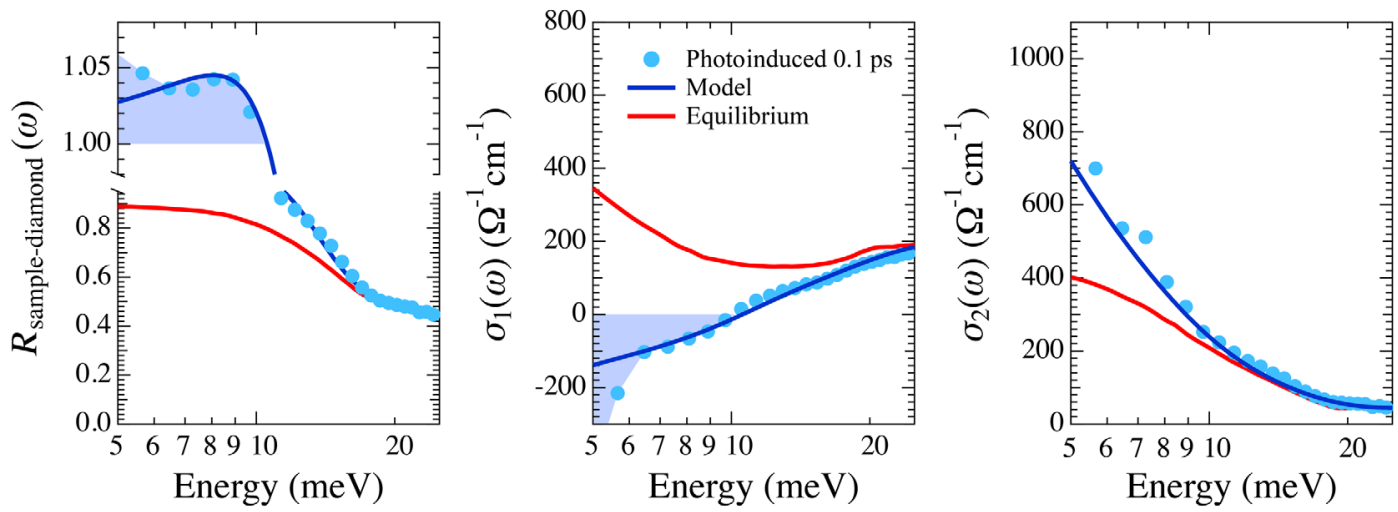

FIG. 4. (a) Reflectivity and complex optical conductivity (sample-diamond interface) of $\mathrm{K}_{3} \mathrm{C}_{60}$ measured at equilibrium (red solid curves) and at the peak of the pump-probe response (light-blue dots) with a pump-pulse duration of 1800 fs. For nonequilibrium systems, we present inferred local quantities at the sample-diamond interface as discussed in Sec. V and Appendix A. (b) Same quantities, measured with a pump-pulse duration of $100 \mathrm{fs}$. The shaded area highlights the frequency range where amplification is observed. All data are taken at $T=100 \mathrm{~K}$ and at a fluence of $4.5 \mathrm{~mJ} / \mathrm{cm}^{2}$. The blue solid curves are the optical conductivity and reflectivity calculated from the theoretical model, taking into account Higgs modulations. The corresponding fit parameters are shown in Table I.

Most interesting, however, are the data at the lowest temperature, $T=25 \mathrm{~K}$, where we again find a lack of amplification. Note that, as shown in Fig. 6(b), the superfluid density of the photoinduced superconducting state at $T=25 \mathrm{~K}$ significantly exceeds that of the low-temperature equilibrium superconductor. This requires borrowing spectral weight from higher energies, beyond that contained in the Drude peak of the normal state, and may be an indication that the superfluid density is near saturation. This offers a possible clue for the lack of amplification at $25 \mathrm{~K}$ : When the order parameter is close to saturation, excitation of the Higgs mode results in oscillations in the pairing gap without significant modulations in the superfluid density, which has little room to oscillate when it is near saturation.

To better understand this last point, it is useful to consider the analogous situation for equilibrium superconductors. The superfluid density $n_{s}=|\psi|^{2}$ and the pairing gap $\Delta$ are independent parameters that can behave very differently from each other. For instance, consider a clean BCS superconductor at low temperatures. Then, $\Delta$ depends on details of the electron-phonon coupling, while the electromagnetic response at frequencies below $2 \Delta$ is given by a London equation in which the superfluid density $n_{s}$ equals the total electron density [55]. In this case, the Higgs mode consists of modulations of the pairing gap $\Delta$, but $n_{s}$ cannot be modulated because all carriers participate in the superconductivity. By contrast, in dirty superconductors, in superconductors with large quantum fluctuations (e.g., due to competing orders), and in superconductors at finite temperature, the superfluid density is strongly reduced from its saturation value, making room for oscillations in $n_{s}$ to occur together with the oscillations in $\Delta$.

\section{COMPARISON OF THEORY AND EXPERIMENT}

The experimental data do not give a direct measurement of the Higgs frequency-in the light-induced superconducting state, the gap measured in $\sigma_{1}(\omega)$ coincides with the 

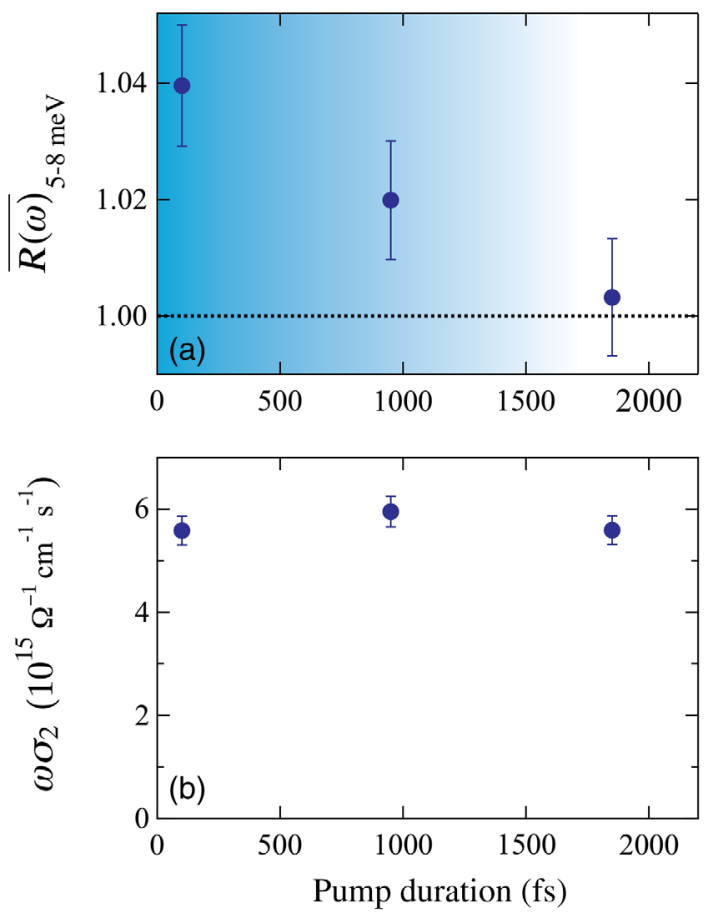

FIG. 5. (a) Inferred local transient reflectivity (samplediamond interface), averaged between 5 and $8 \mathrm{meV}$, as a function of the pump-pulse duration. The blue-shaded region indicates the range of pulse durations where amplification is observed. (b) $\omega \sigma_{2}(\omega \rightarrow 0)=\Lambda_{\mathrm{s}}$, which is proportional to the transient superfluid density, extracted by a low-frequency $1 / \omega$ fit to $\sigma_{2}(\omega)$. All data were taken at $T=100 \mathrm{~K}$ and a constant pump fluence of $4.5 \mathrm{~mJ} / \mathrm{cm}^{2}$.

lower edge of the midinfrared absorption peak of $\mathrm{K}_{3} \mathrm{C}_{60}$, suggesting that the superconducting gap $2 \Delta$ is hidden under the spectral weight of this broad peak. However, there are two independent observations that give consistent estimates for $\omega_{\mathrm{H}}$. First, as argued earlier, the maximum of light amplification occurs slightly below $\omega_{\mathrm{H}} / 2$. The reflectivity measured with the shortest excitation pulse ( $\tau=100 \mathrm{fs}$ ) shown in Fig. 4(a) would then suggest that $\omega_{\mathrm{H}}$ is somewhat bigger than $20 \mathrm{meV} / \hbar$. Second, as mentioned earlier, the onset temperature of the lightinduced superconductivity corresponds to a gap $2 \Delta(T=0)$ of $30 \mathrm{meV} / \hbar$ for the zero-temperature transient superconductor. This value gives an upper bound on $\omega_{\mathrm{H}}$, which tracks $2 \Delta(T)$.

The measured optical conductivity $\sigma(\omega)$ has Higgs modulations built in. In order to make a comparison between theory and experiment, we need to model the optical conductivity of the static superconducting state, $\tilde{\sigma}$. We parametrize $\tilde{\sigma}$ as a sum of Drude and Lorentzian peaks:

$$
\tilde{\sigma}(\omega)=\frac{\Lambda_{\mathrm{s}}}{\gamma_{\mathrm{D}}-i \omega}+\sum_{n=1}^{3} \frac{B_{n} \omega}{i\left(\Omega_{n}^{2}-\omega^{2}\right)+\gamma_{n} \omega} .
$$
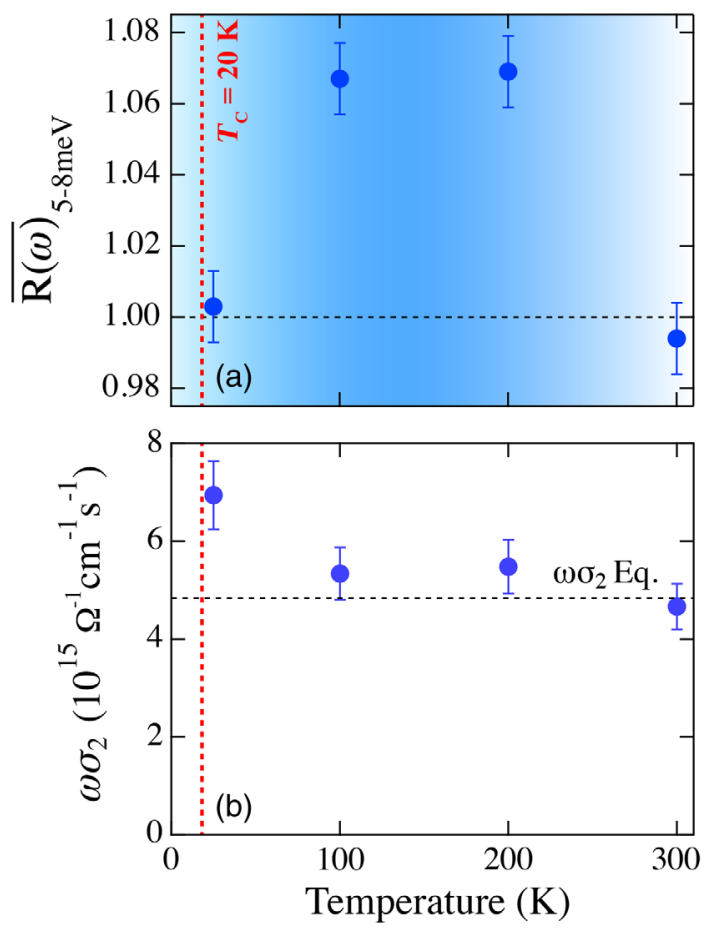

FIG. 6. (a) Inferred local transient reflectivity (sample-diamond interface) averaged between 5 and $8 \mathrm{meV}$, as a function the initial temperature. The blue-shaded region indicates the temperature range where amplification is observed. (b) Temperature dependence of $\omega \sigma_{2}(\omega \rightarrow 0)=\Lambda_{\mathrm{s}}$, which is proportional to the transient superfluid density, extracted by a low-frequency $1 / \omega$ fit to the transient $\sigma_{2}(\omega)$. The red dashed lines indicate the equilibrium superconducting transition temperature $T_{c}=20 \mathrm{~K}$ of $\mathrm{K}_{3} \mathrm{C}_{60}$. All data were taken at a constant pump-pulse duration of $100 \mathrm{fs}$ and a constant pump fluence of approximately $5.5 \mathrm{~mJ} / \mathrm{cm}^{2}$ (which is somewhat larger than the fluence in the data in Figs. 4 and 5).

The Lorentzians represent the broad midinfrared absorption peak in $\mathrm{K}_{3} \mathrm{C}_{60}$ [56]. For simplicity, we assume this peak to be unaffected by the onset of superconductivity, and we fix the parameters $B_{n}, \Omega_{n}$, and $\gamma_{n}$ by fitting to the conductivity of the equilibrium normal state, as discussed in Appendix E. For the light-induced superconducting phase, we maintain the same values for the Lorentzian peaks, while for the Drude peak, we replace $\gamma_{D} \rightarrow 0^{+}$and allow $\Lambda_{\mathrm{s}}$ (which plays the role of the static superfluid density) to vary.

Given the static conductivity $\tilde{\sigma}$, we use the results of Sec. III to model the Higgs amplification phenomenon. We apply Eq. (20) to compute the complex reflection coefficient $r$ at the signal frequency, in which we take $\epsilon(\omega)=\epsilon_{\mathrm{s}}-\tilde{\sigma}(\omega) /\left(i \varepsilon_{0} \omega\right)$. By inverting the Fresnel equation, $r$ is then expressed as an effective optical conductivity $\sigma(\omega)$ (see Appendix D), allowing us to make a comparison of the full complex response of the system.

We find that it is possible to describe the experimental data well by taking $\omega_{\mathrm{H}}=24 \mathrm{meV} / \hbar$. Then, for each value of $\tau$, there are only two parameters we allow to change: 
TABLE I. Model parameters for different pump-pulse widths.

\begin{tabular}{lcc}
\hline \hline$\tau(\mathrm{fs})$ & $\Lambda_{\mathrm{s}} / \Lambda_{s, \mathrm{eq}}$ & $\Lambda_{\mathrm{m}} / \Lambda_{\mathrm{s}}$ \\
\hline 1800 & 1.05 & 0.2 \\
1000 & 1.11 & 0.37 \\
100 & 1.17 & 0.47 \\
\hline \hline
\end{tabular}

the superfluid density $\Lambda_{\mathrm{s}}$ and the amplitude of Higgs modulations, $\Lambda_{\mathrm{m}} / \Lambda_{\mathrm{s}}$. We emphasize that all other parameters in the model are determined in an unbiased manner by comparison with the equilibrium optical properties of the normal state. The blue solid curves in Figs. 4(a) and 4(b) show the complex optical conductivity and the reflectivity of the superconductor, with parameters as chosen in Table I. These results show good agreement with measurements, despite the limited number of fitting parameters used and the simplicity of the model, which does not take into account the full microscopic details of the system. In our fit, we find that the Higgs modulation amplitude increases with decreasing $\tau$, in agreement with the expectation that shorter pulses give rise to more rapid quenches into the superconducting state and therefore to larger Higgs oscillations. In addition, we find that $\Lambda_{\mathrm{s}}$ is slightly larger than its equilibrium value in the normal state $\Lambda_{s, \text { eq }}$. This effect is larger for the shorter pulses. Even though the total pumppulse energy is maintained at a fixed level, the shorter pulses have higher peak intensities and can drive the superconductor more strongly since the pump drives the system nonlinearly. Note that, in order to preserve sum rules, spectral weight is required to transfer from higher energies and may be an indication that the plasmonic peak must be modified in a complete microscopic description of the phenomenon.

The goal of our theoretical analysis is to provide the simplest physical picture of Higgs amplification. Therefore, we limit our discussion to the simplest case, in which the Higgs modulations are assumed to be monochromatic and spatially uniform. In practice, one may expect broadening of the Higgs excitation in frequency, due to its finite lifetime, and in momentum, due to the nonequilibrium character of photoinduced superconductivity. This more comprehensive picture could be obtained by performing frequency- and angle-resolved measurements. However, these experiments would require more advanced instrumentation, including $\mathrm{THz}$ and IR free-electron-laser radiation. Importantly, this advanced instrumentation would allow for the separate measurement of both signal and idler amplification, which would provide direct access to the underlying nonlinear dynamics of the photoinduced superconducting order parameter.

We note that one of the strengths of our model is that it makes falsifiable predictions that discriminate between different interpretations of the observed phenomenology of photoexcited $\mathrm{K}_{3} \mathrm{C}_{60}$, which includes the possibility to distinguish between models where there is an actual, transient, dynamically created, superconducting state, as opposed to other nonequilibrium states. For instance, light amplification has been proposed to occur in the absence of superconductivity [57]. However, in this proposal, symmetry considerations imply that amplification should only occur for reflection at shallow incidence, which is in contrast to our measurements, in which amplification occurs for near-normal incidence. Thus, our observations provide new support that the photoinduced state is indeed superconducting in character.

\section{OUTLOOK}

We envision several potentially interesting applications of the Higgs amplification phenomenon. Of particular interest for quantum information is the possibility for the generation of entangled photon pairs at THz frequencies, as expected from Eq. (1). Properties of the entangled photons may be controlled by tuning the intensity, duration, and angle of incidence of the pump beam.

The notions introduced above can be generalized to the nonlinear dynamics of other kinds of nonequilibrium condensates, including charge and spin density waves, and excitonic condensates.

Systems with several competing orders should exhibit multiple finite-energy collective modes, leading to an additional richness of the order parameter dynamics. The interaction between light and strongly excited collective modes opens a new frontier in the study of light-matter interactions in many-body quantum states.

\section{ACKNOWLEDGMENTS}

We thank R. Averitt, I. Carusotto, J. Faist, M. Hatridge, A. Imamoglu, A. Georges, A. Millis, D. Pekker, and P. Zoller for illuminating discussions. We acknowledge financial support from the European Research Council under the European Union's Seventh Framework Programme (FP7/ 2007-2013)/ERC Grant Agreement No. 319286 (QMAC), the Deutsche Forschungsgemeinschaft via the excellence cluster "The Hamburg Centre for Ultrafast Imaging Structure, Dynamics and Control of Matter at the Atomic Scale" and the priority program SFB925, the National Science Foundation (NSF), the Israel Science Foundation (Grant No. 1803/18), the Harvard-MIT Center for Ultracold Atoms, the DARPA DRINQS program (Grant No. D18AC00014), and the Vannevar Bush Faculty Fellowship. Y. W. is supported by the Postdoctoral Fellowship of the Harvard-MPQ Center for Quantum Optics and the AFOSR-MURI Photonic Quantum Matter (Grant No. FA95501610323). This research used resources of the National Energy Research Scientific Computing Center (NERSC), a U.S. Department of Energy Office of Science User Facility operated under Contract No. DEAC02-05CH11231. D. P. is grateful for the hospitality of 
ITAMP at the Harvard-Smithsonian Center for Astrophysics, and of the Aspen Center for Physics, which is supported by NSF Grant No. PHY-1607611.

M. B. and G. J. contributed equally to this work.

\section{APPENDIX A: ELECTROMAGNETIC RESPONSE OF A SUPERCONDUCTOR WITH HIGGS MODULATIONS}

Consider a superconductor with a time-dependent superfluid density $n_{s}$,

$$
n_{s}(t)=n_{s, 0}+\delta n(t),
$$

where $n_{s, 0}$ is the steady-state value of $n_{s}$ and $\delta n(t)$ is a modulation due to Higgs oscillations. In the main text, we focused on the monochromatic case, $\delta n(t)=$ $2 \delta_{n} \cos \left(\omega_{H} t\right)$, but we now consider a more general situation. When probed by a weak electromagnetic field, the current density in the system can be written as

$$
\begin{aligned}
\mathbf{j}(t)= & \int d t^{\prime} \Pi\left(t-t^{\prime}\right) \mathbf{v}_{s}\left(t^{\prime}\right) \\
& +\int d t^{\prime} d t^{\prime \prime} K\left(t-t^{\prime}, t-t^{\prime \prime}\right) \delta n\left(t^{\prime}\right) \mathbf{v}_{\mathbf{s}}\left(t^{\prime \prime}\right),
\end{aligned}
$$

which is correct to linear order in both $\mathbf{v}_{s}=(\hbar / 2 e) \nabla \theta-\mathbf{A}$ $(\theta$ is the superconducting phase and $\mathbf{A}$ is the vector potential) and the Higgs modulation $\delta n$. Here, for simplicity, we have assumed these fields to be homogeneous in space; otherwise, one must generalize the response functions $\Pi$ and $K$ to include spatial dependence as well. By causality, $\Pi\left(t_{1}\right)$ and $K\left(t_{1}, t_{2}\right)$ vanish unless their arguments are positive.

After Fourier transforming, the relation above becomes

$$
\mathbf{j}(\omega)=\Pi(\omega) \mathbf{v}_{s}(\omega)+\int d \omega^{\prime} K\left(\omega^{\prime}, \omega-\omega^{\prime}\right) \delta n\left(\omega^{\prime}\right) \mathbf{v}_{s}\left(\omega-\omega^{\prime}\right)
$$

Note that, since $\partial_{t} \mathbf{v}_{s}(t)=\mathbf{E}(t), \Pi(\omega)$ is related to the optical conductivity $\sigma(\omega)$ of the steady-state superconductor by $\Pi(\omega)=-i \omega \sigma(\omega)$. Causality implies that the response function $K\left(\omega_{1}, \omega_{2}\right)$ is analytic in the upper half complex plane as a function of each of its two arguments. Hence, it satisfies independent Kramers-Kronig relations with respect to both $\omega_{1}$ and $\omega_{2}$ :

$$
\begin{gathered}
\operatorname{Re} K\left(\omega_{1}, \omega_{2}\right)=\mathcal{P} \frac{1}{\pi} \int_{-\infty}^{\infty} \frac{\operatorname{Im} K\left(\omega_{1}^{\prime}, \omega_{2}\right)}{\omega_{1}^{\prime}-\omega_{1}} d \omega_{1}^{\prime}, \\
\operatorname{Im} K\left(\omega_{1}, \omega_{2}\right)=-\mathcal{P} \frac{1}{\pi} \int_{-\infty}^{\infty} \frac{\operatorname{Re} K\left(\omega_{1}^{\prime}, \omega_{2}\right)}{\omega_{1}^{\prime}-\omega_{1}} d \omega_{1}^{\prime},
\end{gathered}
$$

and similarly for $\omega_{2}$.
One can recover Eq. (4) for $K\left(t-t^{\prime}, t-t^{\prime \prime}\right) \propto$ $\delta\left(t-t^{\prime}\right) \delta\left(t-t^{\prime \prime}\right)$, i.e., by assuming an instantaneous response to the external fields. This procedure is justified in the limit where the external fields vary slowly compared to all microscopic energy scales, and we assume that this is a good starting approximation for the frequencies considered here. Note that this form respects causality and therefore is guaranteed to satisfy the Kramers-Kronig relations. Also note that in our comparison to experiment, dissipation is taken into account in $\Pi(\omega)=-i \omega \sigma(\omega)$, where $\sigma(\omega)$ is chosen as in the discussion following Eq. (27).

\section{APPENDIX B: DETERMINING THE OPTICAL CONDUCTIVITY OF AN EQUIVALENT HOMOGENEOUS MEDIUM}

In the time-resolved experiments, one measures the pump-induced difference in the complex, reflected, electric field $\Delta \tilde{E}_{r}(\omega)$. The "raw" complex reflection coefficient in the photoexcited state $\tilde{r}_{\text {pumped }}(\omega)$ can then be extracted by inverting the following equation:

$$
\frac{\Delta \tilde{E}_{r}(\omega)}{\tilde{E}_{r}^{0}(\omega)}=\frac{\tilde{r}_{\text {pumped }}(\omega)-\tilde{r}_{0}(\omega)}{\tilde{r}_{0}(\omega)},
$$

where $\tilde{r}_{0}(\omega)$ is the unperturbed complex reflection coefficient of $\mathrm{K}_{3} \mathrm{C}_{60}$ known from broadband FTIR measurement and $\tilde{E}_{r}^{0}(\omega)$ is the reflected electric field in the unperturbed state. If the pump light penetrates to a distance which is several times longer than the probe light, one can assume that the probe pulse samples a volume in the material that has been homogeneously transformed by the pump. In this case, it is possible to directly extract the complex-valued optical response functions by inverting the Fresnel equations.

The conditions assumed in the previous paragraph are not correct for the experiments presented here because the penetration depth of the midinfrared pump $(220 \mathrm{~nm})$ is at least 3 times shorter than that of the THz probe $(600-$ $900 \mathrm{~nm}$ ). Nevertheless, it is instructive to see what results for the optical conductivity would be obtained if the assumption is made.

In the first panel of Fig. 7, we show raw data for the reflectivity, in equilibrium and after photoexcitation by pulses of three different lengths. In the second and third panels, we show the values of $\sigma_{1}(\omega)$ and $\sigma_{2}(\omega)$ that one would obtain from the observed reflectivities using the Fresnel equations, assuming optical conductivities independent of distance from the surface.

In contrast, the curves in Fig. 4 were obtained after taking into account that the penetration depth of the probe radiation is longer than that of the pump pulse, using the following approach. As the pump penetrates the material, its intensity is reduced, and it will induce progressively weaker changes in the refractive index of the sample. 
Raw data: no correction for the inhomogeneous optical pump across the probed volume
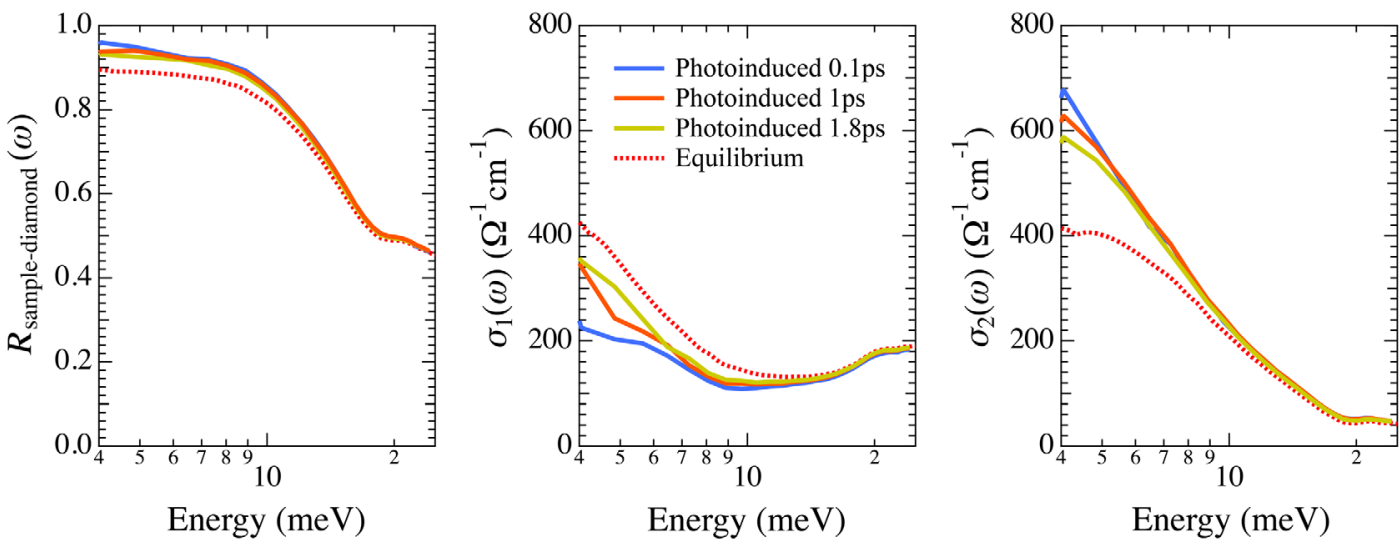

FIG. 7. The first panel shows raw data for the reflectivity (sample-diamond interface), in equilibrium and after photoexcitation by pulses of three different lengths. The second and third panels show the values of $\sigma_{1}(\omega)$ and $\sigma_{2}(\omega)$ that one would obtain from the observed reflectivity via the Fresnel equations, assuming optical conductivities independent of distance from the surface. Note that this neglects the fact that the intensity of the pump-pulse decays as a function of distance from the surface of the sample, and the probe is sampling an inhomogeneously transformed volume.

This situation is modeled by "slicing" the probed thickness of the material into thin layers, where we assume that the pump-induced changes in the refractive index are proportional to the pump intensity in the layer, i.e., $\tilde{n}(\omega, z)=\tilde{n}_{0}(\omega)+\Delta \tilde{n}(\omega) e^{-\alpha z}$, where $n_{0}(\omega)$ is the unperturbed complex refractive index, $\alpha$ is the attenuation coefficient at the pump frequency, and $z$ is the spatial coordinate along the sample thickness.

For each probe frequency $\omega_{i}$, the complex reflection coefficient $\tilde{r}(\Delta \tilde{n})$ of such a multilayer stack is calculated with a characteristic matrix approach [58], keeping $\Delta \tilde{n}$ as a free parameter. As Eq. (B1) directly relates the measured quantity $\Delta \tilde{E}_{r}(\omega) / \tilde{E}_{r}^{0}(\omega)$ to the changes in reflectivity, we can extract $\Delta \tilde{n}$ by minimizing numerically:

$$
\left|\frac{\Delta \tilde{E}_{r}\left(\omega_{i}\right)}{\tilde{E}_{r}^{0}\left(\omega_{i}\right)}-\frac{\tilde{r}\left(\omega_{i}, \Delta \tilde{n}\right)-\tilde{r}_{0}\left(\omega_{i}\right)}{\tilde{r}_{0}\left(\omega_{i}\right)}\right| .
$$

Note that $\Delta \tilde{n}(\omega)$ represents the pump-induced change in the refractive index at the surface, where the pump has not yet been attenuated by the absorption in the material. By taking $\tilde{n}(\omega)=\tilde{n}_{0}(\omega)+\Delta \tilde{n}(\omega)$, one can reconstruct the optical response functions of the material as if it had been homogeneously transformed by the pump.

The blue data points in Fig. 4 show the data processed in this manner to obtain the optical properties applicable to the region closest to the surface, where the excitation pulse is strongest. Specifically, panels in the second and third columns show the effective values of $\sigma_{1}(\omega)$ and $\sigma_{2}(\omega)$ deduced for this region, while the first column shows the reflectivity that would be expected if these values of the optical conductivity were to hold independent of depth. The blue solid curves show values obtained from the theoretical model with Higgs modulation. Comparing
Figs. 4 and 7, we see that while the curves differ in detail, the enhanced optical response for the shortest pulse data, seen in both figures, tends to support our theoretical model. In particular, while we do not actually observe amplification in the raw reflected signal, our analysis suggests that amplification would have been observed if the pump pulse were able to penetrate more deeply into the sample.

\section{APPENDIX C: SIMULATION OF THE POSSIBLE EFFECTS OF SAMPLE HEATING}

In this Appendix, we consider whether the optical properties observed in the experiment could be due to the temperature change of the sample caused by absorption of the pump laser. In the following, we simulate the expected change in optical properties that would arise if the only effect of the midinfrared pump laser consisted of heating up the sample. As discussed in Appendix B, the finite penetration depth of the pump has to be taken into account to obtain the response functions of a homogeneously transformed medium. Note that this also applies in a heating scenario, where the amount of energy absorbed from the pump decays exponentially as a function of distance from the surface [59]. To describe this scenario, we model the energy density deposited by the pump as

$$
E(z)=(1-R) \frac{\mathcal{F}}{d_{\text {pump }}} \exp \left(-\frac{z}{d_{\text {pump }}}\right),
$$

where $R$ is the reflectivity of the sample at the frequency of the pump, $d_{\text {pump }} \approx 0.22 \mu \mathrm{m}$ is the intensity penetration depth of the pump calculated from the unperturbed optical properties as $4 \pi \operatorname{Im}\left(\tilde{n}\left(\lambda_{\text {pump }}\right)\right) / \lambda_{\text {pump }}$, and $\mathcal{F}=4.5 \mathrm{~mJ} / \mathrm{cm}^{2}$ is the excitation fluence. The temperature profile $T_{\mathrm{f}}(z)$ 
Simulated data: pump-induced heating-only scenario after correction for pump-probe mismatch
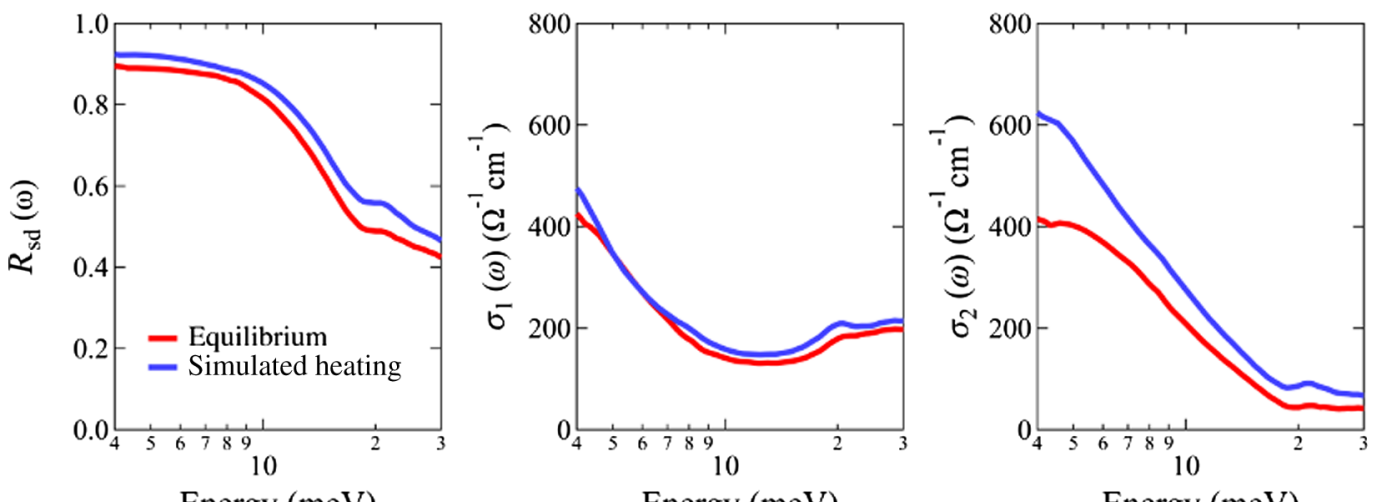

Energy (meV)

Energy $(\mathrm{meV})$

Energy (meV)

FIG. 8. Simulated reflectivity (sample-diamond interface) and real and imaginary parts of the optical conductivity, retrieved using the procedure described in Appendix C for a pump-induced heating-only scenario.

inside the sample is related to the deposited energy density $E(z)$ by the following integral equation:

$$
E(z)=\int_{T_{\mathrm{i}}}^{T_{\mathrm{f}}(z)} C_{p}(T) d T,
$$

where $T_{\mathrm{i}}=100 \mathrm{~K}$ is the initial temperature of the sample and $C_{p}(T)$ is the specific heat of $\mathrm{K}_{3} \mathrm{C}_{60}$ taken from the interpolation of an equilibrium measurement [60]. At the surface of the material, the temperature reaches approximately $280 \mathrm{~K}$ for the fluence used in this work. As heat diffusion happens on much longer timescales [61] than those explored in this work, the temperature profile $T_{\mathrm{f}}(z)$ is assumed to be constant in time.

The spatial distribution of the complex refractive index is then determined via $\tilde{n}(\omega, z)=\tilde{n}\left(\omega, T_{\mathrm{f}}(z)\right)$. The corresponding values of $\tilde{n}(\omega, T)$ are extracted from equilibrium measurements of $\tilde{n}(\omega)$ at $100 \mathrm{~K}, 200 \mathrm{~K}$, and $300 \mathrm{~K}$, with a linear interpolation for intermediate values. We note that the equilibrium optical properties of $\mathrm{K}_{3} \mathrm{C}_{60}$ show a very weak temperature dependence in the range between $100 \mathrm{~K}$ and $300 \mathrm{~K}$ [8].

The complex reflection coefficient of this stratified medium $\tilde{r}_{\mathrm{p}}(\omega)$ is then calculated using a characteristic matrix approach [58]. From this, the quantity that would be measured in a transient $\mathrm{THz}$ reflection experiment, $\Delta \tilde{E}_{r}(\omega) / \tilde{E}_{r}^{0}(\omega)$ [see Eq. (B1)], is then obtained as

$$
\frac{\Delta \tilde{E}_{r}(\omega)}{\tilde{E}_{r}^{0}(\omega)}=\frac{\tilde{r}_{\mathrm{p}}(\omega)-\tilde{r}_{0}(\omega)}{\tilde{r}_{0}(\omega)},
$$

where $\tilde{r}_{0}(\omega)$ is the complex equilibrium reflection coefficient at the base temperature $T=100 \mathrm{~K}$. The result of this calculation is then reprocessed to extract the optical properties of a homogeneously transformed medium using the same method as described in Appendix B. The results are shown in Fig. 8. Importantly, the reconstructed optical properties show very different signatures from those shown in Fig. 4. The reflectivity of the homogeneously transformed medium is only slightly enhanced $(\approx 5 \%)$ with respect to the equilibrium value, and the optical conductivity spectrum shows an increase of the Drude spectral weight in $\sigma_{1}(\omega)$, rather than the opening of an optical gap. Furthermore, while the data reported in Fig. 4 show no change for all probe photon energies greater than about $18 \mathrm{meV}$, the simulated data show changes up to about $30 \mathrm{meV}$. These observations show that the signatures of Higgs-based amplification-such as above-unity reflectivity, a negative $\sigma_{1}(\omega)$, and divergent $\sigma_{2}(\omega)$ —annot be explained in a heating-only scenario.

\section{APPENDIX D: EXPRESSING THE REFLECTIVITY IN TERMS OF A COMPLEX CONDUCTIVITY FUNCTION}

In theory, we compute the complex reflection amplitude $r$, using Eq. (20). To convert this to a conductivity function, we use the Fresnel relation for the reflection amplitude at the interface between two media,

$$
r(\omega)=\frac{\sqrt{\epsilon_{\mathrm{out}}}-i \sqrt{-\epsilon(\omega)}}{\sqrt{\epsilon_{\mathrm{out}}}+i \sqrt{-\epsilon(\omega)}} .
$$

Here, the first medium is diamond, which, to a good approximation, has a frequency-independent dielectric function $\sqrt{\epsilon_{\mathrm{out}}}=2.37$. This relation is inverted,

$$
\epsilon(\omega)=\epsilon_{\text {out }}\left(\frac{1-r(\omega)}{1+r(\omega)}\right)^{2},
$$

in order to obtain the complex conductivity using $\sigma(\omega)=-i \varepsilon_{0} \omega\left[\epsilon(\omega)-\epsilon_{\mathrm{s}}\right]$. 


\section{APPENDIX E: OPTICAL CONDUCTIVITY IN THE NORMAL STATE}

We model the optical conductivity $\tilde{\sigma}(\omega)$ of the equilibrium normal state as the sum of a Drude peak, representing the conduction band, and a sum of three Lorentzians, representing a broad midinfrared absorption peak [56]:

$$
\sigma_{\mathrm{eq}}(\omega)=\frac{\Lambda_{s, \mathrm{eq}}}{\gamma_{\mathrm{D}}-i \omega}+\sum_{n=1}^{3} \frac{B_{n} \omega}{i\left(\Omega_{n}^{2}-\omega^{2}\right)+\gamma_{n} \omega} .
$$

A fit to the optical conductivity of the equilibrium normal state in the measured range $4 \mathrm{meV} / \hbar<\omega<$ $100 \mathrm{meV} / \hbar$ (see Fig. 9) gives the following values: $\Lambda_{s, \mathrm{eq}}=3,470 \Omega^{-1} \mathrm{~cm}^{-1} \mathrm{meV} / \hbar, \quad \gamma_{\mathrm{D}}=3.56 \mathrm{meV} / \hbar$, $B_{1}=18,300 \Omega^{-1} \mathrm{~cm}^{-1} \mathrm{meV} / \hbar, \quad \Omega_{1}=70.4 \mathrm{meV} / \hbar$, $\gamma_{1}=86.6 \mathrm{meV} / \hbar, \quad B_{2}=4,600 \Omega^{-1} \mathrm{~cm}^{-1} \mathrm{meV} / \hbar$, $\Omega_{2}=26.1 \mathrm{meV} / \hbar, \quad \gamma_{2}=34.0 \mathrm{meV} / \hbar, \quad B_{3}=$ 2, $400 \Omega^{-1} \mathrm{~cm}^{-1} \mathrm{meV} / \hbar, \quad \Omega_{3}=102.6 \mathrm{meV} / \hbar, \quad$ and $\gamma_{3}=35.0 \mathrm{meV} / \hbar$.
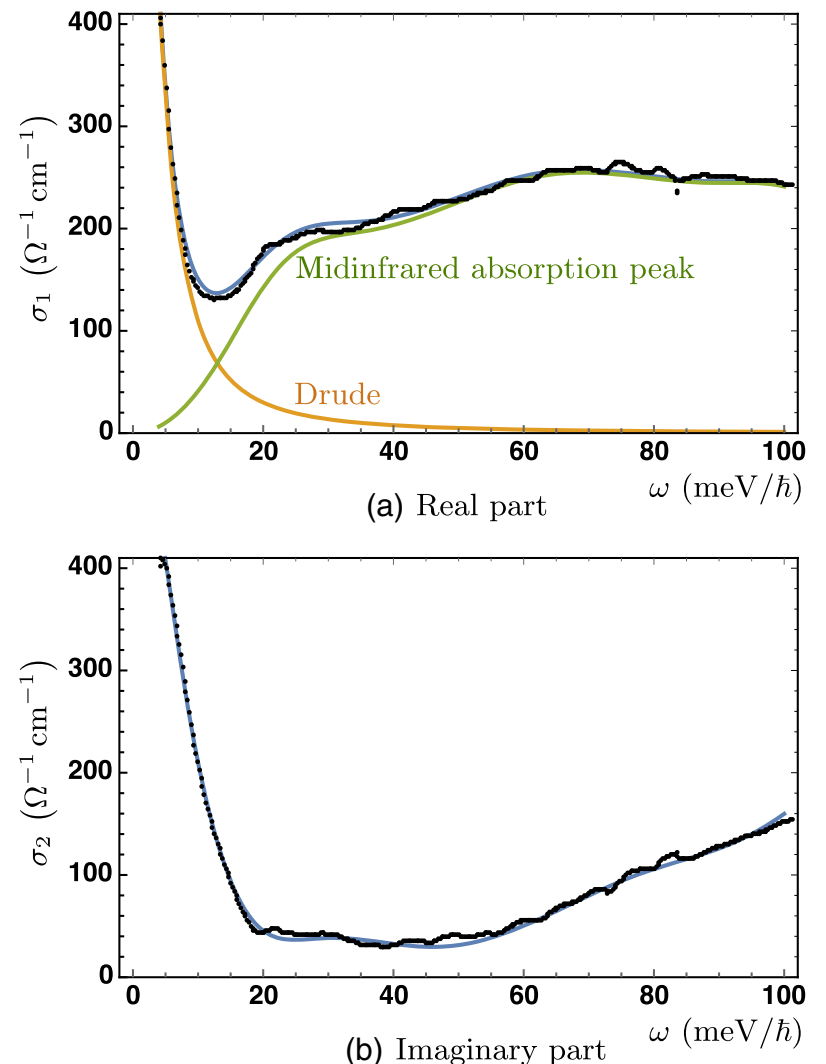

FIG. 9. Optical conductivity of the normal state at equilibrium. Black dots: measured conductivity. Blue curve: fit to a sum of Drude and Lorentzian peaks, Eq. (E1). Orange and green curves: contributions to $\sigma_{1}$ arising from the Drude peak and Lorentzian peaks, respectively. The Lorentzian peaks represent a broad midinfrared absorption peak, which we assume, for simplicity, not to be affected by the onset of superconductivity.
[1] K. Nasu, Photoinduced Phase Transitions (World Scientific, Singapore, 2004).

[2] M. Rini, A. Cavalleri, R. W. Schoenlein, R. López, L. C. Feldman, R. F. Haglund, L. A. Boatner, and T. E. Haynes, Photoinduced Phase Transition in $\mathrm{VO}_{2}$ Nanocrystals: Ultrafast Control of Surface-Plasmon Resonance, Opt. Lett. 30, 558 (2005).

[3] V. R. Morrison, R. P. Chatelain, K. L. Tiwari, A. Hendaoui, A. Bruhács, M. Chaker, and B. J. Siwick, A Photoinduced Metal-Like Phase of Monoclinic $\mathrm{VO}_{2}$ Revealed by Ultrafast Electron Diffraction, Science 346, 445 (2014).

[4] L. Stojchevska, I. Vaskivskyi, T. Mertelj, P. Kusar, D. Svetin, S. Brazovskii, and D. Mihailovic, Ultrafast Switching to a Stable Hidden Quantum State in an Electronic Crystal, Science 344, 177 (2014).

[5] J. Zhang, X. Tan, M. Liu, S. W. Teitelbaum, K. W. Post, F. Jin, K. A. Nelson, D. N. Basov, W. Wu, and R. D. Averitt, Cooperative Photoinduced Metastable Phase Control in Strained Manganite Films, Nat. Mater. 15, 956 (2016).

[6] D. Fausti, R. I. Tobey, N. Dean, S. Kaiser, A Dienst, M. C. Hoffmann, S. Pyon, T. Takayama, H. Takagi, and A. Cavalleri, Light-Induced Superconductivity in a StripeOrdered Cuprate, Science 331, 189 (2011).

[7] D. N. Basov, R. D. Averitt, and D. Hsieh, Towards Properties on Demand in Quantum Materials, Nat. Mater. 16, 1077 (2017).

[8] M. Mitrano, A. Cantaluppi, D. Nicoletti, S. Kaiser, A. Perucchi, S. Lupi, P. Di Pietro, D. Pontiroli, M. Riccò, S. R. Clark et al., Possible Light-Induced Superconductivity in $\mathrm{K}_{3} \mathrm{C}_{60}$ at High Temperature, Nature (London) 530, 461 (2016).

[9] A. Cantaluppi, M. Buzzi, G. Jotzu, D. Nicoletti, M. Mitrano, D. Pontiroli, M. Riccò, A. Perucchi, P. Di Pietro, and A. Cavalleri, Pressure Tuning of Light-Induced Superconductivity in $\mathrm{K}_{3} \mathrm{C}_{60}$, Nat. Phys. 14, 837 (2018).

[10] T. Oka and S. Kitamura, Floquet Engineering of Quantum Materials, Annu. Rev. Condens. Matter Phys. 10, 387 (2019).

[11] J. W. McIver, B. Schulte, F. U. Stein, T. Matsuyama, G. Jotzu, G. Meier, and A. Cavalleri, Light-Induced Anomalous Hall Effect in Graphene, Nat. Phys. 16, 38 (2020).

[12] Y. H. Wang, H. Steinberg, P. Jarillo-Herrero, and N. Gedik, Observation of Floquet-Bloch States on the Surface of a Topological Insulator, Science 342, 453 (2013).

[13] W. Hu, S. Kaiser, D. Nicoletti, C. R. Hunt, I. Gierz, M. C. Hoffmann, M. Le Tacon, T. Loew, B. Keimer, and A. Cavalleri, Optically Enhanced Coherent Transport in $\mathrm{YBa}_{2} \mathrm{Cu}_{3} \mathrm{O}_{6.5}$ by Ultrafast Redistribution of Interlayer Coupling, Nat. Mater. 13, 705 (2014).

[14] R. Mankowsky, A. Subedi, M. Först, S. O. Mariager, M. Chollet, H. T. Lemke, J. S. Robinson, J. M. Glownia, M. P. Minitti, A. Frano, M. Fechner, N. A. Spaldin, T. Loew, B. Keimer, A. Georges, and A. Cavalleri, Nonlinear Lattice Dynamics as a Basis for Enhanced Superconductivity in $\mathrm{YBa}_{2} \mathrm{Cu}_{3} \mathrm{O}_{6.5}$, Nature (London) 516, 71 (2014).

[15] A. Dienst, E. Casandruc, D. Fausti, L. Zhang, M. Eckstein, M. Hoffmann, V. Khanna, N. Dean, M. Gensch, S. Winnerl, 
W. Seidel, S. Pyon, T. Takayama, H. Takagi, and A. Cavalleri, Optical Excitation of Josephson Plasma Solitons in a Cuprate Superconductor, Nat. Mater. 12, 535 (2013).

[16] S. Rajasekaran, E. Casandruc, Y. Laplace, D. Nicoletti, G. D. Gu, S. R. Clark, D. Jaksch, and A. Cavalleri, Parametric Amplification of a Superconducting Plasma Wave, Nat. Phys. 12, 1012 (2016).

[17] R. Matsunaga, Y. I. Hamada, K. Makise, Y. Uzawa, H. Terai, Z. Wang, and R. Shimano, Higgs Amplitude Mode in the BCS Superconductors $\mathrm{Nb}_{1-x} \mathrm{Ti}_{x} \mathrm{~N}$ Induced by Terahertz. Pulse Excitation, Phys. Rev. Lett. 111, 057002 (2013).

[18] R. Matsunaga, N. Tsuji, H. Fujita, A. Sugioka, K. Makise, Y. Uzawa, H. Terai, Z. Wang, H. Aoki, and R. Shimano, Light-Induced Collective Pseudospin Precession Resonating with Higgs Mode in a Superconductor, Science 345, 1145 (2014).

[19] R. Matsunaga, N. Tsuji, K. Makise, H. Terai, H. Aoki, and R. Shimano, Polarization-Resolved Terahertz ThirdHarmonic Generation in a Single-Crystal Superconductor NbN: Dominance of the Higgs Mode beyond the BCS Approximation, Phys. Rev. B 96, 020505(R) (2017).

[20] K. Katsumi, N. Tsuji, Y. I. Hamada, R. Matsunaga, J. Schneeloch, R. D. Zhong, G. D. Gu, H. Aoki, Y. Gallais, and R. Shimano, Higgs Mode in the d-Wave Superconductor $\mathrm{Bi}_{2} \mathrm{Sr}_{2} \mathrm{CaCu}_{2} \mathrm{O}_{8+x}$ Driven by an Intense Terahertz Pulse, Phys. Rev. Lett. 120, 117001 (2018).

[21] R. Sooryakumar and M. V. Klein, Raman Scattering by Superconducting-Gap Excitations and Their Coupling to Charge-Density Waves, Phys. Rev. Lett. 45, 660 (1980).

[22] P. B. Littlewood and C. M. Varma, Amplitude Collective Modes in Superconductors and Their Coupling to ChargeDensity Waves, Phys. Rev. B 26, 4883 (1982).

[23] C. M. Varma, Higgs Boson in Superconductors, J. Low Temp. Phys. 126, 901 (2002).

[24] M.-A. Méasson, Y. Gallais, M. Cazayous, B. Clair, P. Rodière, L. Cario, and A. Sacuto, Amplitude Higgs Mode in the $2 \mathrm{H}-\mathrm{NbSe}_{2}$ Superconductor, Phys. Rev. B 89, 060503(R) (2014).

[25] D. Sherman, B. Pracht, U. S. and Gorshunov, S. Poran, J. Jesudasan, M. Chand, P. Raychaudhuri, M. Swanson, N. Trivedi, A. Auerbach et al., The Higgs Mode in Disordered Superconductors Close to a Quantum Phase Transition, Nat. Phys. 11, 188 (2015).

[26] C. Rüegg, B. Normand, M. Matsumoto, A. Furrer, D. F. McMorrow, K. W. Krämer, H. U. Güdel, S. N. Gvasaliya, H. Mutka, and M. Boehm, Quantum Magnets under Pressure: Controlling Elementary Excitations in $\mathrm{TlCuCl}_{3}$, Phys. Rev. Lett. 100, 205701 (2008).

[27] Y. Ren, Z. Xu, and G. Lupke, Ultrafast Collective Dynamics in the Charge-Density-Wave Conductor $\mathrm{K}_{0.3} \mathrm{MoO}_{3}$, J. Chem. Phys. 120, 4755 (2004).

[28] J. P. Pouget, B. Hennion, C. Escribe-Filippini, and M. Sato, Neutron-Scattering Investigations of the Kohn Anomaly and of the Phase and Amplitude Charge-Density-Wave Excitations of the Blue Bronze $\mathrm{K}_{0.3} \mathrm{MoO}_{3}$, Phys. Rev. B 43, 8421 (1991).

[29] R. Yusupov, T. Mertelj, V. V. Kabanov, S. Brazovskii, P. Kusar, J. H. Chu, I. R. Fisher, and D. Mihailovic, Coherent Dynamics of Macroscopic Electronic Order through a Symmetry Breaking Transition, Nat. Phys. 6, 681 (2010).
[30] M. Endres, T. Fukuhara, D. Pekker, M. Cheneau, and P. Schau $\beta$, C. Gross, E. Demler, S. Kuhr, and I. Bloch, The Higgs Amplitude Mode at the Two-Dimensional SuperfluidMott Insulator Transition, Nature (London) 487, 454 (2012).

[31] U. Bissbort, S. Götze, Y. Li, J. Heinze, J. S. Krauser, M. Weinberg, C. Becker, K. Sengstock, and W. Hofstetter, Detecting the Amplitude Mode of Strongly Interacting Lattice Bosons by Bragg Scattering, Phys. Rev. Lett. 106, 205303 (2011).

[32] S. J. Denny, S. R. Clark, Y. Laplace, A. Cavalleri, and D. Jaksch, Proposed Parametric Cooling of Bilayer Cuprate Superconductors by Terahertz Excitation, Phys. Rev. Lett. 114, 137001 (2015).

[33] D. M. Kennes, E. Y. Wilner, D. R. Reichman, and A. J. Millis, Transient Superconductivity from Electronic Squeezing of Optically Pumped Phonons, Nat. Phys. 13, 479 (2017).

[34] A. Nava, C. Giannetti, A. Georges, E. Tosatti, and M. Fabrizio, Cooling Quasiparticles in $\mathrm{A}_{3} \mathrm{C}_{60}$ Fullerides by Excitonic Mid-infrared Absorption, Nat. Phys. 14, 154 (2018).

[35] M. Babadi, M. Knap, I. Martin, G. Refael, and E. Demler, Theory of Parametrically Amplified Electron-Phonon Superconductivity, Phys. Rev. B 96, 014512 (2017).

[36] R. A. Barankov, L. S. Levitov, and B. Z. Spivak, Collective Rabi Oscillations and Solitons in a Time-Dependent BCS Pairing Problem, Phys. Rev. Lett. 93, 160401 (2004).

[37] E. A. Yuzbashyan, O. Tsyplyatyev, and B. L. Altshuler, Relaxation and Persistent Oscillations of the Order Parameter in Fermionic Condensates, Phys. Rev. Lett. 96, 097005 (2006).

[38] R. A. Barankov and L. S. Levitov, Synchronization in the BCS Pairing Dynamics as a Critical Phenomenon, Phys. Rev. Lett. 96, 230403 (2006).

[39] P. Calabrese, F. H. L. Essler, and M. Fagotti, Quantum Quench in the Transverse Field Ising Chain: I. Time Evolution of Order Parameter Correlators, J. Stat. Mech. (2012) P07016.

[40] A. A. Clerk, M. H. Devoret, S. M. Girvin, F. Marquardt, and R.J. Schoelkopf, Introduction to Quantum Noise, Measurement, and Amplification, Rev. Mod. Phys. 82, 1155 (2010).

[41] M. P. Westig and T. M. Klapwijk, Josephson Parametric Reflection Amplifier with Integrated Directionality, Phys. Rev. Applied 9, 064010 (2018).

[42] D. Podolsky, A. Auerbach, and D. P. Arovas, Visibility of the Amplitude (Higgs) Mode in Condensed Matter, Phys. Rev. B 84, 174522 (2011).

[43] Z. Y. Ou, Efficient Conversion between Photons and between Photon and Atom by Stimulated Emission, Phys. Rev. A 78, 023819 (2008).

[44] H. Y. Hwang, S. Fleischer, N. C. Brandt, B. G. Perkins Jr, M. Liu, K. Fan, A. Sternbach, X. Zhang, R. D. Averitt, and K. A. Nelson, A Review of Non-linear Terahertz Spectroscopy with Ultrashort Tabletop-Laser Pulses, J. Mod. Opt. 62, 1447 (2015).

[45] M. Graf, G. Scalari, D. Hofstetter, J. Faist, H. Beere, E. Linfield, D. Ritchie, and G. Davies, Terahertz Range Quantum Well Infrared Photodetector, Appl. Phys. Lett. 84, 475 (2004). 
[46] D. C. Mattis and J. Bardeen, Theory of the Anomalous Skin Effect in Normal and Superconducting Metals, Phys. Rev. 111, 412 (1958).

[47] Y. Wang, D. Podolsky, and E. A. Demler, Higgs Amplification in Fluctuating Superconductors (to be published).

[48] A. F. Volkov and S. M. Kogan, Collisionless Relaxation of the Energy Gap in Superconductors, J. Exp. Theor. Phys. 38, 1018 (1974).

[49] M. H. S. Amin, E. V. Bezuglyi, A. S. Kijko, and A. N. Omelyanchouk, Wigner Distribution Function Formalism for Superconductors and Collisionless Dynamics of the Superconducting Order Parameter, Low Temp. Phys. 30, 661 (2004).

[50] M. H. Szymańska, B. D. Simons, and K. Burnett, Dynamics of the BCS-BEC Crossover in a Degenerate Fermi Gas, Phys. Rev. Lett. 94, 170402 (2005).

[51] V. Gurarie, Nonequilibrium Dynamics of Weakly and Strongly Paired Superconductors, Phys. Rev. Lett. 103, 075301 (2009).

[52] E. A. Yuzbashyan, M. Dzero, V. Gurarie, and M. S. Foster, Quantum Quench Phase Diagrams of an s-wave BCS-BEC Condensate, Phys. Rev. A 91, 033628 (2015).

[53] A. Cartella, T. F. Nova, M. Fechner, R. Merlin, and A. Cavalleri, Parametric Amplification of Optical Phonons, Proc. Natl. Acad. Sci. U.S.A. 115, 12148 (2018).

[54] H. Y. Liu, I. Gierz, J. C. Petersen, S. Kaiser, A. Simoncig, A. L. Cavalieri, C. Cacho, I. C. E. Turcu, E. Springate, F. Frassetto, L. Poletto, S. S. Dhesi, Z.-A. Xu, T. Cuk,
R. Merlin, and A. Cavalleri, Possible Observation of Parametrically Amplified Coherent Phasons in $\mathrm{K}_{0.3} \mathrm{MoO}_{3}$ Using Time-Resolved Extreme-Ultraviolet Angle-Resolved Photoemission Spectroscopy, Phys. Rev. B 88, 045104 (2013).

[55] A. A. Abrikosov, L. P. Gorkov, I. E. Dzyaloshinski, and R. A. Silverman, Methods of Quantum Field Theory in Statistical Physics, Dover Books on Physics (Dover Publications, New York, 2012).

[56] M. J. Rice and H.-Y. Choi, Charged-Phonon Absorption in Doped $\mathrm{C}_{60}$, Phys. Rev. B 45, 10173 (1992).

[57] G. Chiriacò, A. J. Millis, and I. L. Aleiner, Transient Superconductivity without Superconductivity, Phys. Rev. B 98, 220510(R) (2018).

[58] M. Born and E. Wolf, Principles of Optics, 4th ed. (Pergamon Press, New York, 1970).

[59] H. Niwa, N. Yoshikawa, K. Tomari, R. Matsunaga, D. Song, H. Eisaki, and R. Shimano, Light-Induced Nonequilibrium Response of the Superconducting Cuprate $\mathrm{La}_{2-x} \mathrm{Sr}_{x} \mathrm{CuO}_{4}$, Phys. Rev. B 100, 104507 (2019).

[60] K. Allen and F. Hellman, Specific Heat of $\mathrm{C}_{60}$ and $\mathrm{K}_{3} \mathrm{C}_{60}$ Thin Films for $T=6-400 \mathrm{~K}$, Phys. Rev. B 60, 11765 (1999).

[61] O. Andersson, A. Soldatov, and B. Sundqvist, Thermal Conductivity of $\mathrm{C}_{60}$ at Pressures up to $1 \mathrm{GPa}$ and Temperatures in the 50-300 K Range, Phys. Rev. B 54, 3093 (1996). 